\title{
REVISIÓN DEL GÉNERO HARRAPHIDIA STEINMANN, 1963 CON LA DESCRIPCIÓN DE DOS NUEVAS ESPECIES DE LA PENÍNSULA IBÉRICA Y DE MARRUECOS (INSECTA, RAPHIDIOPTERA)
}

\author{
V. J. Monserrat * y D. Papenberg **
}

\begin{abstract}
RESUMEN
Se realiza una revisión taxonómica del género Harraphidia Steinmann, 1963. Hasta la fecha este género incluía dos especies: su especie tipo Harraphidia harpyia Steinmann, 1963, conocida de Marruecos, y Harraphidia laufferi (Navás, 1915), conocida de la Península Ibérica. Para esta última especie se reconsidera la validez del subgénero Flavoraphidia n. stat. H. Aspöck \& U. Aspöck, 1968 como subgénero de Harraphidia y se propone Harraphidia (Flavoraphidia) laufferi n. comb. y Harraphidia (Harraphidia) harpyia Steinmann, 1963 n. comb. como nuevas combinaciones. Harraphidia (Harraphidia) subdesertica Monserrat \& Papenberg n. sp. y Harraphidia (Harraphidia) divergens Monserrat \& Papenberg n. sp. se describen como nuevas especies de España y de Marruecos. Se anotan todas las referencias bibliográficas existentes sobre cada una de ellas, se incluye una diagnosis y una clave de identificación de estos subgéneros y especies y se describen nuevos datos sobre su morfología externa, genital, biología, fenología y distribución. Se amplia en este orden de insectos la exigencia, comúnmente aceptada, de que requieren temperaturas inferiores o próximas a $0^{\circ} \mathrm{C}$ durante su desarrollo pre-imaginal, confirmándose la presencia de especies en el S./S.E. Ibérico que se desarrollan en medios donde la temperatura anual no desciende de $8^{\circ} \mathrm{C}$. Se realiza un somero análisis biogeográfico sobre la distribución de estas especies en base a presunto flujo bi-direccional de elementos entre Europa y África durante el Eoceno y el Mioceno.

Palabras clave: Raphidioptera, Harraphidia, Taxonomía, Faunística, Morfología, España, Marruecos.
\end{abstract}

\section{SUMMARY}

A Revision of Genus Harraphidia Steinmann, 1963 with the description of two new species from the Iberian Peninsula and Morocco (Insecta, Raphidioptera)

A taxonomical review of the genus Harraphidia Steinmann, 1963 is made. Up to now this genus included two species: its type species Harraphidia harpyia Steinmann, 1963, known from Morocco, and Harraphidia laufferi (Navás, 1915), known from the Iberian Peninsula. For this last species the subgenus Flavoraphidia H. Aspöck \& U. Aspöck, 1968 is reconsidered and two new combinations Harraphidia (Flavoraphidia) laufferi n. comb. and Harraphidia (Harraphidia) harpyia Steinmann, 1963 n. comb. are proposed. Two new species: Harraphidia (Harraphidia) subdesertica Monserrat \&

\footnotetext{
* Departamento de Zoología y Antropología Física. Facultad de Biología, Universidad Complutense, E-28040 Madrid, Spain.
} E-mail:*artmad@bio.ucm.es;** davpap@telefonica.net 
Papenberg n. sp. and Harraphidia (Harraphidia) divergens Monserrat \& Papenberg n. sp. are described from Spain and Morocco. All known bibliographical references to these species are reviewed, and a diagnosis and a subgenera -and species- keys are included and new data on their external morphology, genitalia, biology, phaenology and geographical distribution are given. The generally accepted view that this order of insects requires spending a period at temperature below or close to $0^{\circ} \mathrm{C}$ during its preimaginal development is amplified to $8^{\circ} \mathrm{C}$ as the minimum annual temperature for the pre-imaginal development in some S./S.E. Iberian species. A brief bio-geographical analysis of the distribution of these species is also undertaken, suggesting a possible bidirectional flow of elements between Europe and Africa during the Eocene and the Miocene.

Key Words: Raphidioptera, Harraphidia, Taxonomy, Faunistics, Morphology, Spain, Morocco.

\section{Introducción}

Hasta el presente, y en función de los datos existentes en la bibliografía y en los que hemos venido recientemente obteniendo, la fauna de Rafidiópteros (Insecta, Raphidioptera) de la Península Ibérica consta de ocho géneros pertenecientes a dos familias (Raphidiidae e Inocelliidae) e incluye trece especies (más una que describimos en esta contribución) que poseen una distribución mayoritariamente circunscrita a los dos tercios meridionales de la península. Es curioso y sorprendente el hecho de que salvo Atlantoraphidia maculicollis y puntuales datos de tres especies de los géneros Xanthostigma y Phaeostigma, son casi inexistentes los datos y las citas de este orden de insectos en todo el tercio septentrional de la Península Ibérica, incluyendo toda la Cornisa Cantábrica y Pirineos, y este hecho sugiere un origen faunístico mayoritariamente norte-africano de la fauna rafidiopterológica ibérica, y por ello que no puede abordarse su estudio sin tener presente la fauna del norte de África.

Como primera contribución a la revisión de los rafidiópteros de la Península Ibérica presentamos en este artículo la revisión del género Harraphidia Steinmann, 1963, que hasta el momento constaba de dos especies: su especie tipo Harraphidia harpyia Steinmann, 1963, conocida de Marruecos y Harraphidia laufferi (Navás, 1915), conocida del centro y sur de la Península Ibérica y que, como conjunto, representa un ejemplo de una distribución biogeográfica marcadamente bético-rifeña. Como es sabido, desde el punto de vista biogeográfico, la Región Bético-Rifeña integra el norte de Marruecos y gran parte de las provincias españolas de Cádiz y Málaga, pudiendo algunos de sus elementos extenderse a otras zonas peninsulares meridionales adyacentes.
La fauna de rafidiópteros de la Región BéticoRifeña ha recibido poca atención en relación con otras áreas biogeográficas, sobre todo en lo que respecta al Continente Africano. Sobre éste último y desde los trabajos de Lestage (1922, 1928) sólo Steinmann (1963), U. Aspöck \& H. Aspöck (1969), H. Aspöck et al. (1983), H. Aspöck (1990), Monserrat et al. (1991) y H. Aspöck \& Hölzel (1996) han aportado datos faunísticos, morfológicos o descripciones de nuevos taxa en esta zona.

En el norte de África se han citado cuatro especies pertenecientes a cuatro géneros: Africoraphidia spilonota (Navás, 1915) de Argelia y Marruecos, Harraphidia harpyia Steinmann, 1963 y Mauroraphidia maghrebina H. Aspöck, U. Aspöck \& Rausch, 1983 de Marruecos y Fibla (Fibla) peyerimhoffi (Navás, 1919) de Argelia, Túnez y Marruecos. En la Península Ibérica se han citado dos de estos géneros con las especies Harraphidia laufferi (Navás, 1915) y Fibla (Fibla) hesperica Navás, 1915, ambas mayoritariamente presentes en el centro y sur de España y Portugal.

En relación al género que tratamos en esta contribución, desde que Steinmann (1963) describió el género Harraphidia y su especie tipo, Harraphidia harpyia, sólo se ha incrementado con la especie anteriormente citada Harraphidia laufferi, que descrita dentro del género Raphidia por Navás (1915a) fue trascrita a este género por H. Aspöck (1990).

\section{Material y Método}

Para la revisión del género Harraphidia se ha estudiado el material existente en las diferentes colecciones entomológicas que se anotan a continuación:

(NHM): Natural History Museum (Londres, Reino Unido). (EEZ): $\quad$ Estación Experimental del Zaidín (Granada, España). 
(MNCN): Museo Nacional de Ciencias Naturales (Madrid, España).

(UAM): Universidad Autónoma (Madrid, España).

(VM): Colección Víctor J. Monserrat, Universidad Complutense (Madrid, España).

Tanto para el género Harraphidia, como para cada una de sus dos especies anteriormente conocidas y de sus sinonimias actualmente aceptadas, se recopilan, cronológicamente ordenadas, todas las referencias bibliográficas existentes, anotando los autores de estas citas, su fecha de publicación y la página donde se aporta información sobre sus alas (a), biología (bio), inclusión en claves (c), descripción original (d), distribución geográfica (dis), generalidades (gen), identificación errónea (ie), larva (l), morfología (mf), pupa (p), referencia (rf), sinonimia ( $\sin$ ), listado (lst), taxonomía $(\mathrm{tx})$, genitalia del macho $\left(\sigma^{7}\right)$ o genitalia de la hembra ( $(9)$. En cualquier caso, la inclusión de estas citas es informativa y no siempre implica la total asunción de sus contenidos, especialmente para la información dada por autores que no utilizaban la genitalia para la identificación de las especies, pero es obvio que mucha de esta información sí se ha tenido en cuenta, dada la autoridad de los autores que la han aportado y que merecen toda nuestra confianza.

Posteriormente de cada especie se aportan los datos de su morfología externa, segmentos genitales, biología, fenología y distribución así como un apartado de discusión, donde se comentan los datos que hemos considerado necesarios, seguidos del material estudiado.

Para la exposición del material estudiado los datos se exponen alfabética y cronológicamente ordenados, indicándose por este orden: el país, provincia y localidad de captura, coordenadas UTM o geográficas, altitud en $\mathrm{m}$, fecha de captura, planta sobre la que los ejemplares han sido capturados, número de $\sigma^{7} \sigma^{7}$ y de $\odot$ $\odot$ estudiados, recolector $\mathrm{e}$ institución a la que el material pertenece según las siglas anteriormente anotadas. Con la intención de no reiterar información, en la enumeración de las localidades de captura, éstas se separarán por un punto cuando correspondan a diferentes localidades y por una coma cuando todo o parte de la ubicación o nombre de la localidad ya haya sido anotada en los ejemplares citados inmediatamente antes, pormenorizándose sólo las diferencias en su fecha de captura, número de ejemplares, planta soporte, recolector o institución. En esta relación del material estudiado se señalan con un asterisco $(*)$ aquellos ejemplares elegidos para realizar las figuras de la genitalia masculina $\left(\mathrm{O}^{*}\right)$ o femenina $\left({ }^{*}\right)$ y los dibujos de las alas $\left(\mathrm{a}^{*}\right)$.

Aunque existen datos en la morfología externa que ayudan a la identificación de las especies de este género y así los utilizamos en las claves, el estudio de la genitalia es necesario para la segura y correcta identificación de lo ejemplares. Para ello se ha procedido a la separación de los últimos cuatro segmentos del abdomen mediante disección. Si el material estaba conservado en seco se ha hidratado previamente durante 24 horas por inmersión en agua. Posteriormente se han aclarado estos últimos segmentos con hidróxido potásico al $10 \%$ para acceder a las estructuras genitales, que en el caso de la genitalia interna de las $ᄋ$ 우 se han teñido con negro de clorazol para visualizar sus estructuras. Una vez realizado este proceso hemos introducido la genitalia en glicerina en un porta excavado para su observación mediante un estereo-microscopio LEICA GZ-4. Los dibujos se realizaron utilizando el programa de diseño vectorial Corel Draw 10.

Para la realización de los mapas de distribución de las especies, se ha utilizado el mismo programa anteriormente citado $\mathrm{y}$, no sólo se ha tenido en cuenta el material estudiado por nosotros, sino también todas aquellas citas que nos resultan incuestionables o absolutamente solventes en función de los autores que las han dado.

\section{Revisión del género Harraphidia}

Iniciamos este apartado con la recopilación bibliográfica de las citas referentes a este género, seguido de la diagnosis de su morfología externa, genitalia, biología, fenología y distribución general, así como la discusión de estos datos en relación a la posición taxonómica de alguna de sus especies. En un segundo apartado aportamos la clave de especies utilizando caracteres sencillos fácilmente observables, seguido un tercer apartado con la revisión de cada una de ellas siguiendo la ordenación y exposición de los datos anteriormente anotados. Finalizamos con un último apartado con algunas consideraciones biogeográficas.

\section{1: EL GÉNERO HARRAPHIDIA}

Harraphidia Steinmann, 1963: 188 (d, a), 186 (c), [especie tipo por designación original: Harraphidia harpyia Steinmann, 1963].

Considerado como subgénero de Raphidia Linnaeus, 1758: H. Aspöck \& U. Aspöck, 1968: 63 (tx). U. Aspöck \& H. 
Aspöck, 1969: 168 (tx). H. Aspöck et al., 1975: 371 (1). H. Aspöck et al., 1980b: 78 (p). H. Aspöck et al., 1980a: 87 (dis).

Considerado como género:

Harraphidia Steinmann, 1963: 188 (d, a), 186 (c). Buresch \& Popov, 1973: 155 (rf). H. Aspöck, 1986: 25-26 (dis). H. Aspöck et al., 1989: 89 (dis). H. Aspöck, 1990: 50 (lst), 53 (tx), 54-55 (dis). H. Aspöck et al., 1991: - tomo I: 350 (a, mf, ơ ), 54, 76, 351 (bio), 351, 552, 555, 559 (dis), 107, 111, 115, 351 (tx) 117, 351 ( 우) 351 (l) - tomo II: 424 (dis). Oswald \& Penny, 1991: 64 (lst). U. Aspöck \& H. Aspöck, 1994: 39 (tx). H. Aspöck \& Hölzel, 1996: 38 (gen), 44 (dis). H. Aspöck et al., 2001: 19, 50 (tx), 50 (dis). H. Aspöck, 2002: 38 (1).

= Flavoraphidia H. Aspöck \& U. Aspöck, 1968: 61 (d, a, O', ㅇ, $\mathrm{mf}, \mathrm{tx}$ ), [especie tipo por designación original: Raphidia laufferi Navás, 1915].

Considerado como subgénero de Raphidia Linnaeus, 1758: U. Aspöck \& H. Aspöck, 1969: 168 (dis). H. Aspöck et al., 1975: 371 (1). H. Aspöck et al., 1980a: 87 (dis, gen), 86 (tx, sin). Oswald \& Penny, 1991: 63 (lst).

El género Harraphidia fue descrito por Steinmann (1963) en base a una especie nueva que presentaba una peculiaridad en la venación del ala anterior y que consiste en la existencia de dos venas transversales, una entre la Costa y el Radio y otra entre la Subcosta y el Radio (fig. 1), cuando lo habitual en este orden es una única vena entre la Subcosta y el Radio. Creó para dicha especie el género monotípico Harraphidia (de "Harr" = caliente en árabe) formado por la especie Harraphidia harpyia. Este género sólo se ha incrementado con la especie Harraphidia laufferi, que descrita dentro del género Raphidia por Navás (1915a) fue considerada posteriormente por $\mathrm{H}$. Aspöck y U. Aspöck (1968) como especie tipo del subgénero Flavoraphidia $\mathrm{H}$. Aspöck y U. Aspöck, 1968 y fue posteriormente trascrita al género Harraphidia por H. Aspöck (1990). Ninguna otra especie ha sido relacionada con el género que tratamos y veremos más adelante la problemática relación de $H$. laufferi con la otra especie de este género Harraphidia y la existencia de otras dos nuevas especies pertenecientes a este género.

MORFOLOGÍA EXTERNA: El género incluye especies de tamaño pequeño. La longitud del ala anterior es de 5.5-8.0 $\mathrm{mm}$ en los $\sigma^{7} \sigma^{7}$ y 6.5-8.5 $\mathrm{mm}$ en las 우 우. La cabeza es de coloración negra, ovalada, estrechándose fuertemente hacia su región basal $\mathrm{y}$ presentando un dibujo con bastante relieve.

El pronoto es delgado, pardo oscuro o alternándose el color pardo y el negro según las especies, pudiendo aparecer un dibujo amarillo o pardo. La venación alar es parda, apareciendo en ocasiones venas total o parcialmente amarillas. Es característico en la mayoría de las especies de este género la aparición de una vena transversal supletoria entre la Costa y el Radio antes del pterostigma y de forma esporádica otra entre la Subcosta y el Radio en las alas anteriores (figs. 1, 2, 3) caracteres que no presenta $H$. laufferi (fig. 4). El pterostigma es de tamaño medio, amarillo, está atravesado por una vena, y presenta tras él una vena apical (figs. 1-4). En el ala anterior, la parte distal del sector del radio tiene una vena bifurcada y otra sin bifurcar o ambas aparecen bifurcadas (figs. 1-4). La parte basal de la Mediana anterior del ala posterior transcurre como vena transversal (figs. 1-4).

Segmentos Genitales: En los $0^{7} \sigma^{\top}$ el $8^{\circ}$ esternito es bastante más corto que el $8^{\circ}$ terguito, estando íntimamente ligado al $9^{\circ}$ segmento (figs. 5-12). El $9^{\circ}$ terguito se alarga ventralmente, fusionándose con el $9^{\circ}$ esternito y siendo muy estrecho en la zona central de este alargamiento (figs. 5, 7, 9, 11). El $9^{\circ}$ esternito puede ser relativamente ancho (fig. 12) o muy estrecho en forma de una fina banda (fig. 8). Los gonocoxitos se extienden en dirección dorsoventral, presentan el apodema del estilo largo y bien marcado (figs. 5, 7, 9, 11), en su parte más dorsal existe una pequeña expansión de forma triangular (figs. $5,7,9,11$ ) y su región ventral es grande sin ningún tipo de proceso y su base es marcadamente convexa y está débilmente abombada presentando un margen anterior en forma de banda bien esclerotizada (figs. 5-12). Los estilos son bifurcados con un ápice en dirección dorso-caudal y otro en dirección antero-ventral (figs. 5, 7, 9, 11). El ectoprocto es alargado y romo (figs. 5, 7, 9, 11). La hipovalva está formada por un par de escleritos alargados, rectos, paralelos y próximos entre sí, en forma de banda que en su parte basal son más finos (figs. 6, 8, 10), salvo en $H$. laufferi que están más separados, son más sinuosos y más arqueados y portan lobulaciones externas (fig. 12) y en las dos especies nuevas que se describen existen formaciones membranosas portadoras de dentículos asociadas lateralmente a los citados escleritos de la hipovalva (figs. 8,10 ) que por su constancia parecen tener valor taxonómico. Faltan los parámeros y no se ha localizado gonarco. El hipandrio interno es grande (fig. 12) y existe un endofalo membranoso evaginable (figs. 11, 12).

En las $\$$ \% el $7^{\circ}$ esternito es alargado con el borde caudal convexo (fig. 17). El $8^{\circ}$ terguito presenta el borde antero-ventral convexo (fig. 18), estrechándose ventralmente. No hay una genitalia 

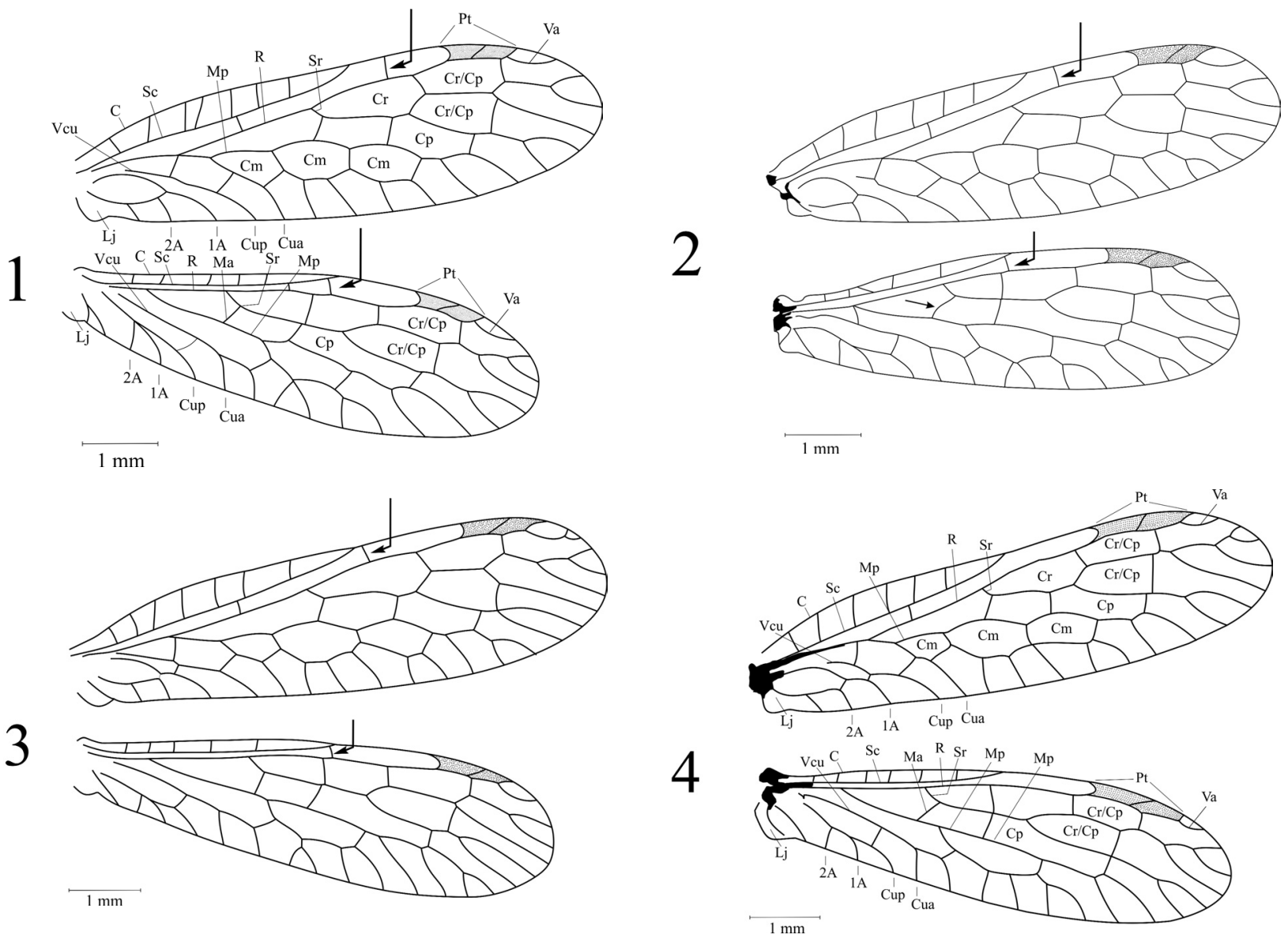

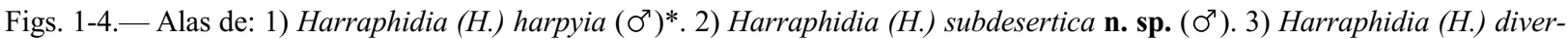
gens n. sp. ( $\left.\sigma^{7}\right)$. 4) Harraphidia (F.) laufferi ( ()$^{*}$. 1A: $1^{\mathrm{a}}$ vena anal, 2A: $2^{\mathrm{a}}$ vena anal, C: costal, Cm: celda media, Cp: celda postigmal, Cr: celda radial, Cua: cúbito anterior, Cup: cúbito posterior, Lj: lóbulo yugal, Ma: mediana anterior, Mp: mediana posterior, Pt: pterostigma, R: radio, Sc: subcostal, Sr: sector del radio, Va: vena apical, Vcu: vena cubital. * De H. Aspöck et al. (1991).

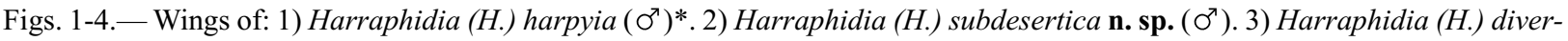
gens n. sp. $\left(\sigma^{7}\right)$. 4) Harraphidia (F.) laufferi ( ()$^{*}$. 1A: anal vein 1, 2A: anal vein 2, C: costal, Cm: median cell, Cp: postigmal cell, Cr: radial cell, Cua: anterior cubitus, Cup: posterior cubitus, $\mathrm{Lj}$ : yugal lobe, Ma: anterior median, Mp: posterior median, Pt: pterostigma, R: radius, Sc: subcostal, Sr: radial sector, Va: apical vein, Vcu: cubital vein. * From H. Aspöck et al. (1991).

externa delimitada. Respecto a la genitalia interna, el atrio de la bolsa copuladora es membranoso y está unido al sáculo al no diferenciarse un ductus saculi (figs. 20-22). El ductus receptaculi es un conducto muy fino de longitud y diámetro variable, pudiendo ser corto, grueso y festoneado (fig. 21) o muy largo, delgado y en forma de ovillo (figs. 20, 22), surge de la zona media y ventral del sáculo (figs. 20,21), salvo en $H$. laufferi que se origina en su extremo anterior (fig. 22). El receptáculo seminal es pequeño, tenue y delicado con el extremo basal piriforme y está levemente estrangulado en su zona basal (figs. 20,21) y su conducto presenta varios lóbulos verrugosos en la zona próxima a desembocar en él (figs. 20, 21), salvo en H. laufferi cuyo receptáculo seminal es helicoidal, más robusto, sin estrangulamiento basal y su conducto carece de lóbulos verrugosos en la zona próxima a desembocar en él (fig. 22). Las glándulas anejas son largas y filiformes presentando su extremo algo dilatado (figs. 20,21).

Biología: Poco se conoce sobre la biología de sus especies. Los imagos parecen tener preferencia por colonizar biotopos mediterráneos xerotermos 
de muy diferentes tipos y han sido preferentemente hallados sobre vegetación arbórea y arbustiva asociada a estos medios mediterráneos, más escasamente sobre microfanerófitos o sobre vegetación herbácea. Su distribución altitudinal global comprende entre los 0-1.800 m, en la Península ibérica alcanza cotas más bajas y se han hallado ejemplares entre 0-1.600 m, mientras que en Marruecos su distribución es exclusivamente montana (800$1.800 \mathrm{~m}$ ). El número de individuos en las poblaciones puede ser frecuentemente alto y sugerimos en alguna especie cierta capacidad de agregación y quizás de posible canibalismo.

La presencia de, al menos, dos especies de este género en zonas térmicas o marcadamente xero-térmicas (con escasa altitud sobre el nivel del mar), en particular en el SE árido de la Península Ibérica (figs. 25, 26), que son zonas donde o no, o difícil o muy excepcionalmente se alcanzan temperaturas inferiores a $0^{\circ} \mathrm{C}$ y que refleja la Tabla 1 (donde anotamos el número de días con temperaturas inferiores a $0^{\circ} \mathrm{C}$, a lo largo de varios años, en diferentes estaciones próximas o en las zonas donde hemos hallado ejemplares) pone en duda, cuestiona, o al menos resulta excepcional, uno de los requerimientos ampliamente aceptados en este orden de insectos sobre la necesidad de temperaturas próximas o por debajo de $0^{\circ} \mathrm{C}$ durante el desarrollo de su larva y su paso a pupa. H. Aspöck (1998) y H. Aspöck et al. (1991) anotaban que el frío invernal es un requisito fundamental en las larvas de este orden para que prosiga el desarrollo y que si se mantienen larvas que deberían pupar en la primavera siguiente duran- te el invierno a temperatura ambiente de un laboratorio, permanecen activas, se alimentan, mudan, pero jamás pasan a estadio de prepupa y que aunque se desconoce si esto es así en todos los casos, en algunas especies se requiere un choque de temperaturas frías aunque dure sólo unos pocos días para inducir la entrada en estado de prepupa. También $\mathrm{H}$. Aspöck (2000) anota que es necesario pasar un periodo de temperaturas bajas (alrededor de $\operatorname{los} 0^{\circ} \mathrm{C}$ o inferior, tolerando temperaturas de hasta $-20^{\circ} \mathrm{C}$ ) para poder pasar a la fase de pupa o el desarrollo hacia el imago y U. Aspöck \& H. Aspöck (1994) mencionan que según todos los estudios realizados por ellos, todas las rafidias actuales necesitan pasar una fase de temperaturas bajas durante su desarrollo para desarrollarse de larva a pupa o de forma poco frecuente de pupa a imago. También este condicionante térmico ha servido de argumentos a diversas teorías sobre la distribución geográfica de muchos de sus taxa (H. Aspöck et al., 1991; U. Aspöck \& H. Aspöck, 1994; H. Aspöck, 2000, 2002).

Este condicionante en la necesidad de temperaturas próximas o inferiores a $0^{\circ} \mathrm{C}$ para su desarrollo no parece cumplirse en, al menos, dos de las especies del género que hemos tratado y parece ser, dentro de este orden de insectos, un hecho bastante excepcional. Sólo Kovarik et al. (1991) que estudiaron la especie americana Agulla bicolor (Albarda, 1891) afirmaban que no necesita frío para iniciar la pupación y en base a esto H. Aspöck (2002, 2003) comentaba que es posible que en algunas especies una corta y leve bajada de las temperaturas sea suficiente. Según anotamos ahora, en

Tabla 1.- Datos recabados del Servicio Nacional de Meteorología sobre su toma de datos en diferentes estaciones meteorológicas existentes en la zona de estudio donde queda anotado el número de días al año ( ) con temperaturas inferiores a $0^{\circ} \mathrm{C}$ a lo largo de varios años de toma de datos $(\mathrm{N})$.

Table 1.- Data obtained from Servicio Nacional de Meteorología of Spain, on temperature records taken in different meteorological stations in the study area, where the number of days per year ( ) with temperatures below $0^{\circ} \mathrm{C}$, along several years $(\mathrm{N})$, are noted.

\begin{tabular}{lccccc}
\hline Estación & Código & Altitud & Longitud & Latitud & N \\
\hline Alcalá de los Gazules & $5980 \mathrm{U}$ & $28 \mathrm{~m}$ & 0543202 & 362323 & $12(5,2,1,1,5)$ \\
Osuna & 5998 & $260 \mathrm{~m}$ & 0506172 & 371400 & $9(0)$ \\
Castellar de la Frontera & 60230 & $260 \mathrm{~m}$ & 0527022 & 361835 & $11(18,1)$ \\
Tarifa & 6001 & $41 \mathrm{~m}$ & 0535522 & 360053 & $56(0)$ \\
Nijar & 6327 & $356 \mathrm{~m}$ & 0212172 & 365800 & $30(1,1,1,2)$ \\
El Ejido & $6291 \mathrm{I}$ & $50 \mathrm{~m}$ & 0245512 & 364222 & $7(0)$ \\
El Ejido de Dalias & 6292 & $50 \mathrm{~m}$ & 0245172 & 364700 & $14(0)$ \\
\hline
\end{tabular}



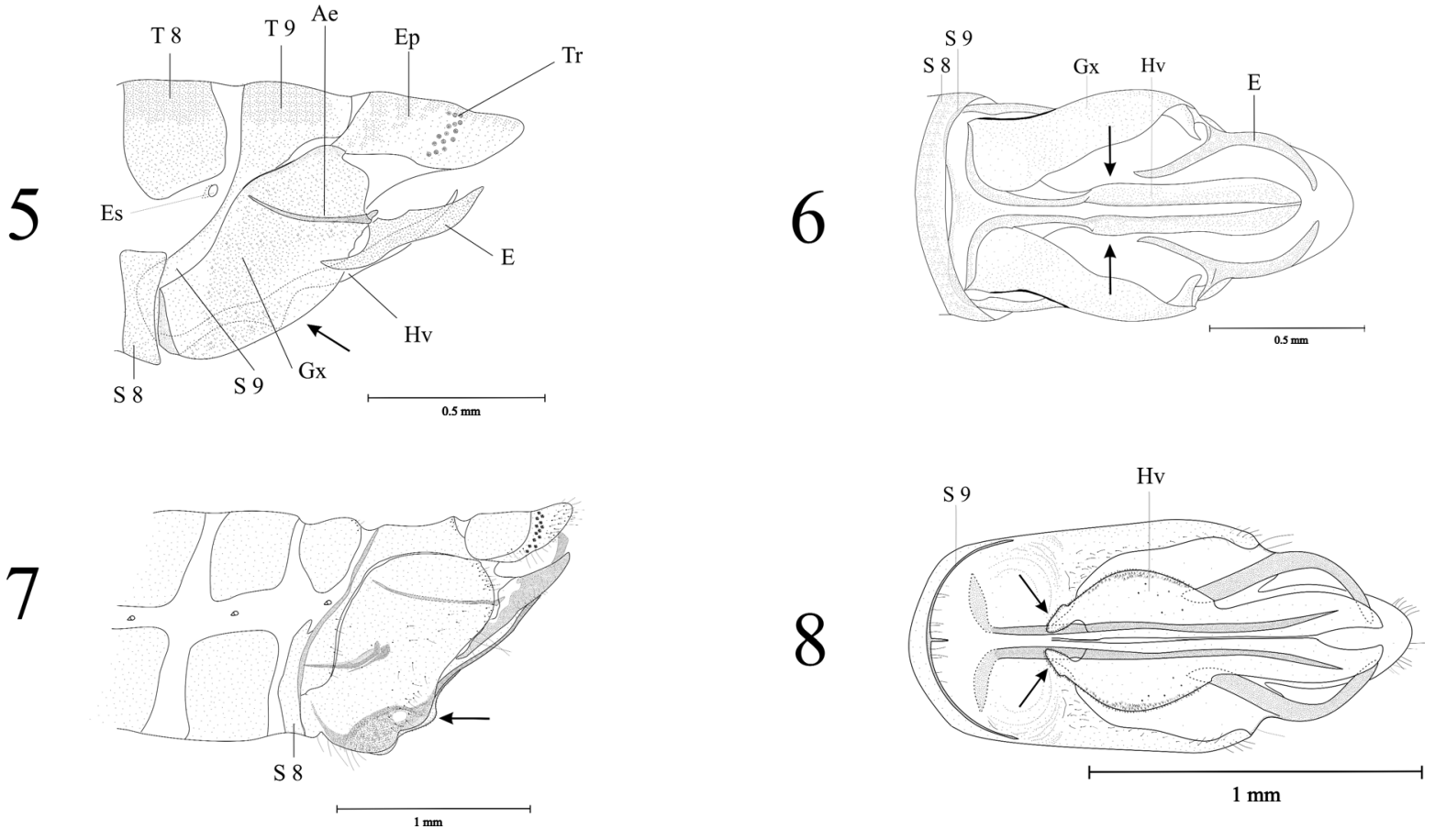

9
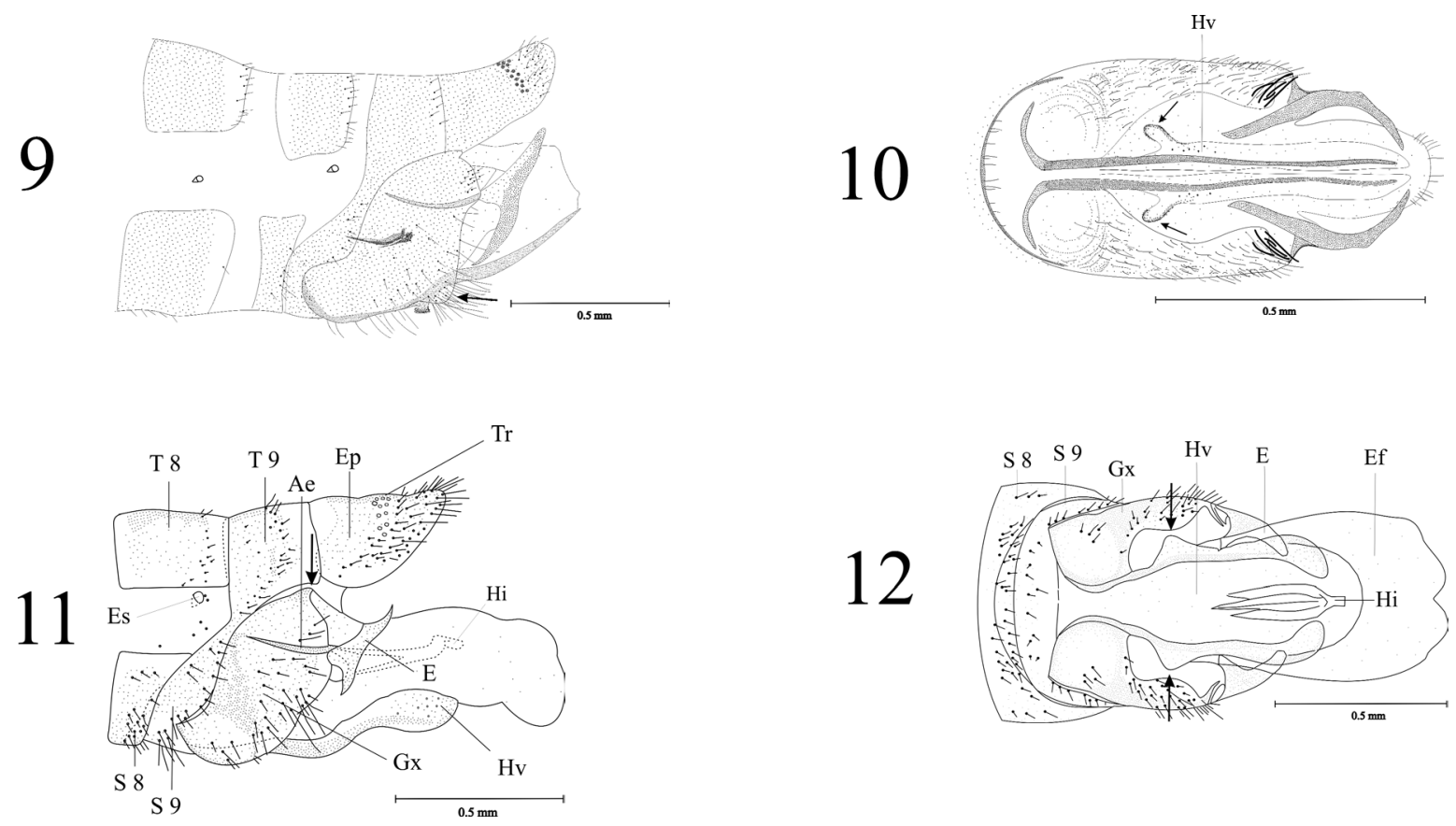

Figs. 5-12.- Genitalia ( $\left.\sigma^{7}\right)$ en vista lateral y ventral de: 5-6) Harraphidia (H.) harpyia*. 7-8) Harraphidia (H.) subdesertica n. sp. 910) Harraphidia (H.) divergens n. sp. 11-12) Harraphidia (F.) laufferi*. Ae: apodema del estilo, E: estilo, Ef: endofalo, Ep: ectoprocto, Es: espiráculo, Hi: hipandrio, Hv: hipovalva, Gx: gonocoxito, S: esternito, T: terguito, Tr: tricobotrios. * De H. Aspöck et al. (1991).

Figs. 5-12.- $\sigma^{7}$ Genitalia in lateral and ventral view of: 5-6) Harraphidia (H.) harpyia*. 7-8) Harraphidia (H.) subdesertica n. sp. 910) Harraphidia (H.) divergens n. sp. 11-12) Harraphidia (F.) laufferi*. Ae: stilus apodeme, E: stilus, Ef: endofalus, Ep: ectoproct, Es: espircle, Hi: hipandrium, Hv: hipovalve, Gx: gonocoxite, S: esternite, T: terguite, Tr: trichobotria. * From H. Aspöck et al. (1991). 


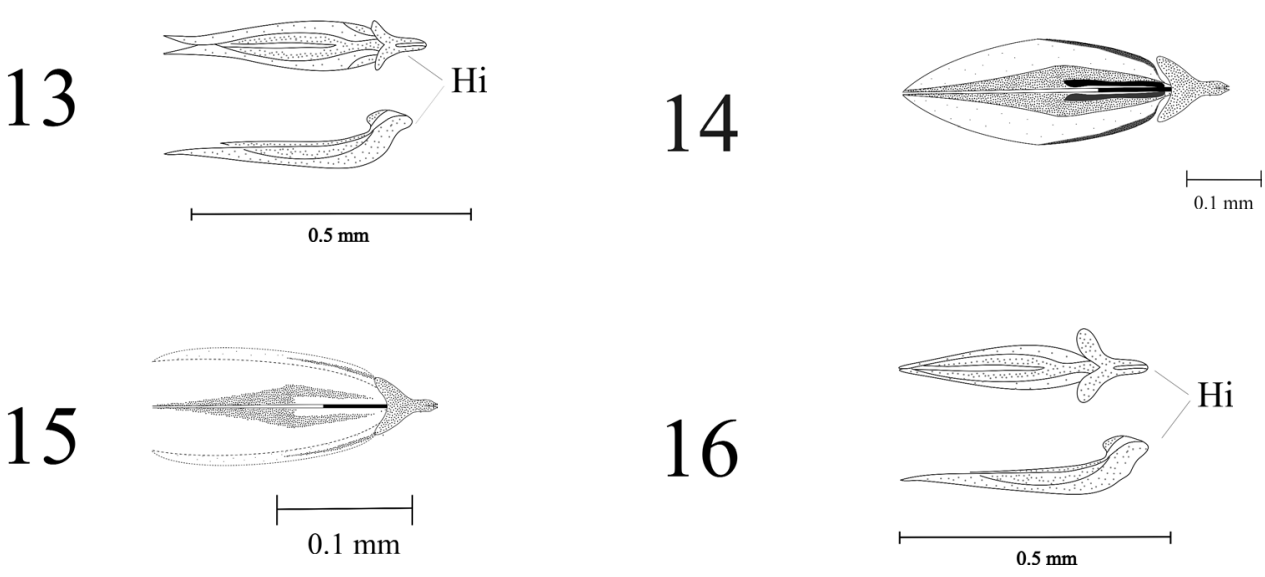

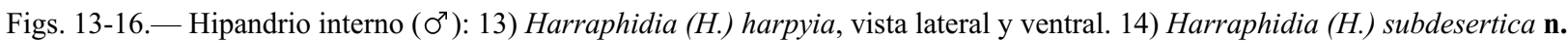
sp., vista ventral*. 15) Harraphidia (H.) divergens n. sp., vista ventral. 16) Harraphidia (F.) laufferi, vista lateral y ventral. * De H. Aspöck et al. (1991).

Figs. 13-16. - Hipandrium internum ( $\left.\sigma^{7}\right)$ : 13) Harraphidia (H.) harpyia, lateral and ventral view. 14) Harraphidia (H.) subdesertica n. sp. ventral view*. 15) Harraphidia (H.) divergens n. sp., ventral view. 16) Harraphidia (F.) laufferi, lateral and ventral view. * From H. Aspöck et al. (1991).

muchas de las zonas donde habitan dos de las especies ibéricas que tratamos, puede asegurarse que su desarrollo pre-imaginal se realiza a temperaturas superiores a $0^{\circ} \mathrm{C}$ (Tabla 1) e incluso, como hemos comprobado para algunas localidades (por ejemplo en Tarifa), a temperaturas mínimas anuales superiores a $8^{\circ} \mathrm{C}$. Parece pues que estas dos especies ibéricas poseen una evidente adaptación a colonizar zonas más térmicas donde no se registran temperaturas próximas o inferiores a $0^{\circ} \mathrm{C}$ y según lo sugerido por H. Aspöck $(2002,2003)$ también parece que en estas dos especies es suficiente una leve disminución de la temperatura en estos meses más fríos del año para proseguir su desarrollo.

Las larvas sólo son conocidas en $H$. laufferi y $H$. harpyia diferenciándose bien unas de otras (figs. 23 , 24). Probablemente son edáficas, las hemos hallado en hojarasca de alcornoque, jaras, pinos y musgos y en la bibliografía anotada se han citado desarrollos larvarios de al menos dos años, en los que la última hibernación transcurre en fase de pupa (al menos en $H$. harpyia), estadio en la que se mantiene entre 6 y 10 meses.

FENOLOGÍA: La actividad de los imagos es monovoltina, estacionaria (mayoritariamente primaveral) y varia según la latitud y la altitud, trans- curriendo durante los meses de Marzo-Julio en las poblaciones ibéricas y entre Mayo-Julio en las marroquíes.

DISTRIBUCIÓN: Citado, en principio, del centro y sur de la Península Ibérica y noroeste de África. Tras la exposición del siguiente apartado veremos que Harraphidia s. str. es un elemento exclusiva y típicamente bético-rifeño.

CONSIDERACIONES TAXONÓMICAS PREVIAS: Tras haber expuesto la diagnosis del género Harraphidia y la descripción general de su morfología externa y de su genitalia masculina y femenina, ha podido observarse la permanente excepción que representa Harraphidia laufferi respecto al resto, y resulta evidente que esta especie posee caracteres morfológicos muy peculiares y distintos a que presentan las otras tres especies consideradas y su ubicación dentro de él resulta problemática según anteriormente mencionábamos.

También hemos indicado que originariamente descrita dentro del género Raphidia por Navás (1915b) Harraphidia laufferi fue considerada posteriormente por H. Aspöck y U. Aspöck (1968) como especie tipo del subgénero Flavoraphidia $\mathrm{H}$. Aspöck y U. Aspöck, 1968 y que fue posteriormente trascrita al género Harraphidia por $\mathrm{H}$. 
Aspöck (1990), y es por ello por lo que aquí la hemos considerado.

Son muchas las diferencias mencionadas, y alguna más que se comentará más adelante, que existen entre Harraphidia laufferi respecto a las otras tres especies, no sólo en la genitalia, sino en su morfología externa y su venación alar dentro de un orden de insectos morfológicamente tan uniforme y homogéneo donde escasean este tipo de diferencias de morfología externa entre sus numerosos géneros y, de hecho, H. Aspöck \& U. Aspöck (1968) llegaron incluso a establecer una clasificación genérica del orden con la existencia de dos únicos géneros: Raphidia para la familia Raphidiidae e Inocellia para la familia Inocelliidae y $\mathrm{H}$. Aspöck et al. (1991) mencionan la excepcionalidad de la venación alar de Harraphidia harpyia dentro de todos los Raphidiidae, diferencias que comparten y mantienen con las otras dos especies que citamos respecto a Harraphidia laufferi. Pero, al margen de la venación, son sobre todo aspectos en la genitalia masculina y femenina anteriormente anotados, que hacen a esta especie merecedora de ser diferenciada taxonómicamente de las otras tres especies.

Por todo ello, nos vemos obligados a reconsiderar el subgénero Flavoraphidia H. Aspöck \& U. Aspöck, 1968 para Harraphidia laufferi y mantenerlo con la categoría de subgénero, manteniendo las otras tres especies conocidas asociadas dentro del subgénero Harraphidia s. str., según anotamos en el siguiente listado:

\section{Género Harraphidia Steinmann, 1963}

Especie tipo: Harraphidia harpyia Steinmann, 1963 por designación original (Steinmann, 1963: 188).

\section{Subgénero Harraphidia Steinmann, 1963}

Especie tipo por monotipia: Harraphidia (Harraphidia) harpyia Steinmann, 1963 y por designación original subsecuente (Steinmann, 1963: 188).

Especies conocidas:

Harraphidia (Harraphidia) harpyia Steinmann, 1963 n. comb.

Harraphidia (Harraphidia) subdesertica Monserrat \& Papenberg n. sp.

Harraphidia (Harraphidia) divergens Monserrat \& Papenberg n. sp.

Subgénero Flavoraphidia H. Aspöck \& U. Aspöck, 1968 n. stat.

Especie tipo: Raphidia laufferi Navás, 1915, designada por H. Aspöck \& U. Aspöck, 1968: 61.
Fue descrito como subgénero de Raphidia Linnaeus, 1758 (H. Aspöck \& U. Aspöck, 1968: 61) y aquí considerado como subgénero de Harraphidia.

Especies conocidas:

Harraphidia (Flavoraphidia) laufferi (Navás, 1915)

n. comb.

Como diagnósticos del subgénero Flavoraphidia frente a Harraphidia s. str. destacamos parte de los caracteres ya anotados por H. Aspöck \& U. Aspöck (1968) y H. Aspöck et al. (1980a) junto a otros que ahora anotamos y que mejor pueden definirlo:

Venación alar sin una vena transversal supletoria entre la Costa y el Radio antes del pterostigma, ni entre la Subcosta y el Radio en las alas anteriores (fig. 4 frente a figs. 1-3). Ausencia de una o dos venas trasversales en el campo subcostal de las alas posteriores (fig. 4 frente a figs. 1$3)$. En el $\sigma^{7}$ la hipovalva está formada por un par de escleritos separados, sinuosos, arqueados y que portan lobulaciones externas (fig. 12 frente a figs. $6,8,10$ ). El hipandrio interno es grande (fig. 16) y el endofalo está presente. En la $\$$ el ductus receptaculi surge del extremo anterior del sáculo (fig. 22 frente a figs. 20,21). El receptáculo seminal es helicoidal, robusto, sin estrangulamiento basal y su conducto carece de lóbulos verrugosos en la zona próxima a desembocar en él (fig. 22 frente a figs. 20, 21). Las glándulas anejas son largas y filiformes presentando una dilatación en el extremo.

Distribución: El subgénero Flavoraphidia es un elemento ibérico conocido del centro y sur de la Península Ibérica (fig. 26) de distribución geográfica distinta al subgénero Harraphidia que posee una distribución típicamente bético-rifeña (fig. 25).

\section{2: CLAVE DE IDENTIFICACIÓN DE LAS ESPECIES DE HARRAPHIDIA}

$$
\sigma^{7} 0^{x}
$$

1 Ala anterior con una vena transversal suplementaria entre la Costa y el Radio (figs. 1-3) Subgénero Harraphidia ...... 2

- Ala anterior sin esta vena transversal suplementaria (fig. 4) Subgénero Flavoraphidia, única especie: ............ Harraphidia (Flavoraphidia) laufferi n. comb.

2 Hipovalva sin procesos o ensanchamientos laterales (fig. 6) ............Harraphidia (Harraphidia) harpyia n. comb. Hipovalva con procesos o ensanchamientos laterales (fig.

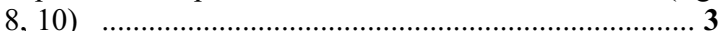


3 Hipovalva ensanchada en su zona media y con procesos laterales orientados hacia la línea media (fig. 8) ............... Harraphidia (Harraphidia) subdesertica n. sp.

- Hipovalva no ensanchada en su zona media y con procesos laterales orientados hacia afuera (fig. 10) Harraphidia (Harraphidia) divergens $\mathbf{n} . \mathbf{s p}$.

우 우

( $\odot$ de Harraphidia (H.) divergens n. sp. desconocida, pero iría al paso 2)

1 Ala anterior con una vena transversal suplementaria entre la Costa y el Radio (figs. 1-3) Subgénero Harraphidia ...... 2 Ala anterior sin esta vena transversal suplementaria (fig. 4) Subgénero Flavoraphidia, única especie: ..Harraphidia (Flavoraphidia) laufferi n. comb.

2 Ductus receptaculi extremadamente largo, delgado y enrollado en forma de ovillo (fig. 20) Harraphidia (Harraphidia) harpyia $\mathbf{n}$. comb. Ductus receptaculi corto, grueso, no enrollado en forma de ovillo, sino levemente festoneado (fig. 21) Harraphidia (Harraphidia) subdesertica n. sp.

\section{3: LAS ESPECIES DEL GÉNERO HARRAPHIDIA}

Harraphidia (Harraphidia) harpyia Steinmann, 1963 n. comb.

Harraphidia harpyia Steinmann, 1963

Harraphidia harpyia Steinmann, 1963: 188 (d), (dis). H. Aspöck \& U. Aspöck, 1969: 35 (dis, tx). H. Aspöck, 1990: 52 (bio), 48, 52, 53 (dis), 50 (1st), 51 (1). H. Aspöck et al., 1991, - tomo I: 63, 353, 594 (a), 148 - 149 (c), 350-352 (mf), 350 ( ○'), 351 (우), 351-352 (l), 351 - 352, 555, 586, 605 (dis), 351 - 352 (bio), 351 (tx), 352 (gen), - tomo II: 52 (mf), 74 (a), $174\left(\sigma^{7}\right), 354$ (1), 486 (dis). H. Aspöck \& Hölzel, 1996: 44 (dis). H. Aspöck et al., 2001: 51 (dis), 343 (tx).

Raphidia (Harraphidia) harpyia (Steinmann, 1963)

H. Aspöck \& U. Aspöck, 1968: 63 (tx). U. Aspöck \& H. Aspöck, 1969: 166 (gen), 168 (dis), 163, 164 (○', 우). H Aspöck \& U. Aspöck, 1971: 39 ( 우). Monserrat et al., 1991: 102 (dis, ver Harraphidia divergens $\mathbf{n} . \mathbf{s p}$.).

Raphidia maculicollis Stephens, 1836

Esben-Petersen, 1931: 6 (dis, ie).

Material Estudiado: MARRUECOS: Al Hoceima: Tagsut,

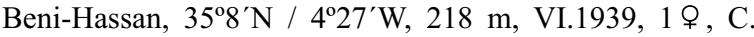
Bolivar (MNCN). Chaouen: Xauen, Cordillera del Rif, $35^{\circ} 10^{\prime} \mathrm{N} / 5^{\circ} 16^{\prime} \mathrm{W}, 600 \mathrm{~m}, 1$ 우, M. Escalera (MNCN), Zoco Telata, Ketama, 35⒉ $24^{\prime} \mathrm{N} / 5^{\circ} 30^{\prime} \mathrm{W}, 1.715$ m, VI.1932, 2 우 우, M. Escalera (MNCN).

MORFOLOGÍA EXTERNA: Es una especie de tamaño pequeño. La longitud del ala anterior mide entre 6,5-7,5 $\mathrm{mm}$ en el $\sigma^{7} \mathrm{y}$ entre 7-8,5 $\mathrm{mm}$ en la 우. El clípeo y el labro son pardos. El escapo es pardo, el pedicelo amarillo ocre y el flagelo pardo. El prono- to en su parte anterior es pardo oscuro y en la posterior pardo-negruzco con un dibujo pardo en la región más caudal. Las coxas I son pardas y anteriormente amarillas, las coxas II y III son pardas oscuras y los trocánteres II y III son pardos, el resto de los segmentos son amarillo sucio. La venación alar (fig. 1) es, casi en su totalidad, parda. La Costa, Subcosta y el tercio proximal del Radio y partes de algunas venas aisladas de la base del ala son amarillas. El ala anterior presenta una vena transversal suplementaria entre la Costa y el Radio, en posición proximal al pterostigma (fig. 1) y de forma más ocasional existe otra vena entre la Subcosta y el Radio. La coloración de los terguitos y esternitos es pardo oscura, presentando estos últimos un borde caudal amarillo. El $5^{\circ}$ al $7^{\circ}$ esternito de la 우 tienen manchas amarillas. Los gonocoxitos del $\sigma^{\gamma}$ en $\mathrm{su}$ parte ventral son amarillos.

SEGMENTOS GENITALES: En el $\sigma^{\top}$ los escleritos de la hipovalva son rectilíneos, sin presentar procesos laterales, y en su parte final convergen y se unen (fig. 6). El hipandrio interno es grande (fig. 13). En la ㅇ el intersegmento $7 / 8$ es corto, membranoso con grandes pliegues (fig. 17). El ductus receptaculi surge de la zona media ventral del sáculo (fig. 20) siendo muy largo y formando un ovillo. El ductus receptaculi presenta unos lóbulos verrugosos característicos en su región terminal próximos al receptáculo seminal (fig. 20).

BiologíA: El desarrollo probablemente se realice sobre el sustrato edáfico. Hay registros y hemos hallado larvas y adultos en sustratos y fanerófitos, mayoritariamente Quercus, Olea, Pinus, Celtis y Juniperus y nanofanerofitos, principalmente genistas, pistaceas y otros arbustos en zonas de bosques de coníferas, principalmente Pinus. Para sus larvas (fig. 23) se han citado periodos de desarrollo de 2 años (H. Aspöck et al., 1991). La última hibernación transcurre en fase de pupa. La pupación tiene lugar en los meses VI-X. Los biotopos que ocupa son xerotermos. Parece una especie asociada a medios montanos, su distribución altitudinal comprende $800-1.800 \mathrm{~m}$. Respecto a la larva se diferencia de la de $H$. (F.) laufferi por la ausencia de una mancha parda, aislada dentro de las bandas laterales pálidas (fig. 23). Los registros son muy localizados y las densidades de población pueden ser puntualmente altas.

FENOLOGÍA: Registros entre los meses de JunioJulio.

DistriBUCiÓN: Marruecos (fig. 25). Elemento faunístico estacionario, monocéntrico, mauritánico. 

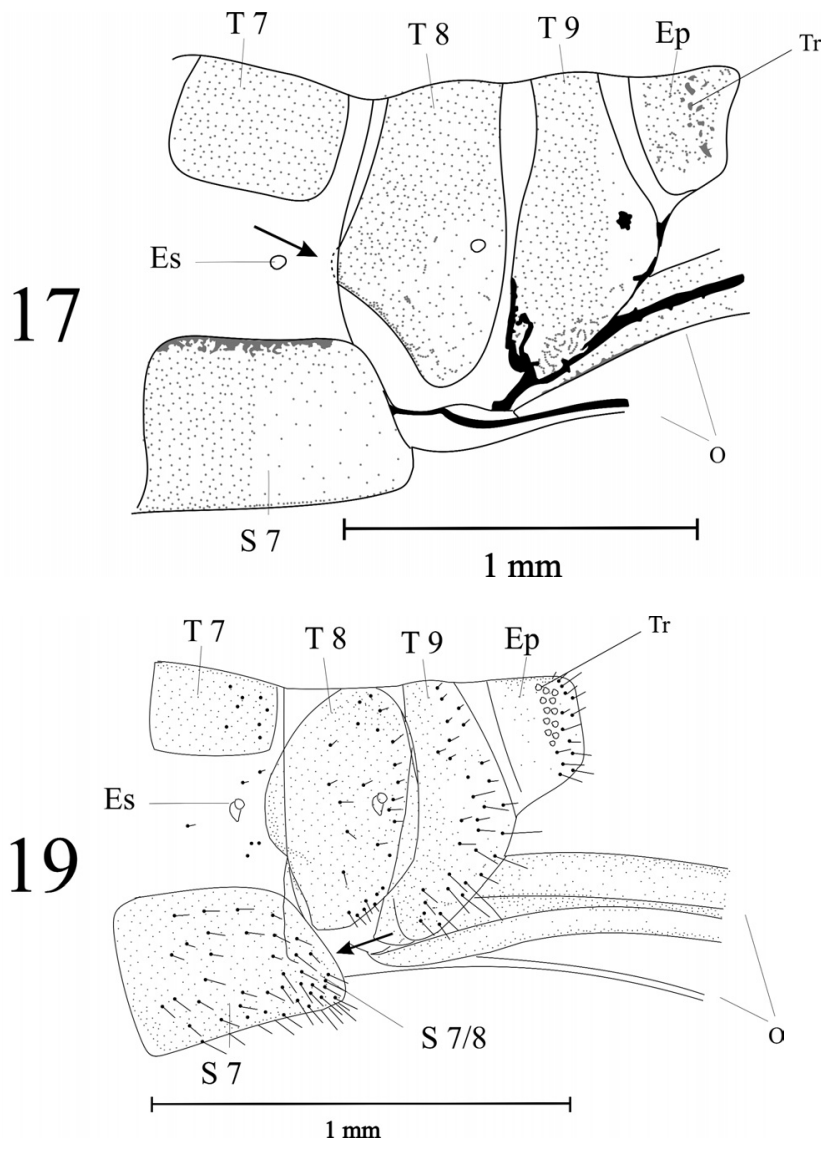

Su distribución es escasamente conocida. La mayoría de las citas proceden de las cadenas montañosas de El Rif y del Medio y Alto Atlas. Es posible que la especie también colonice áreas montañosas de Argelia y Túnez (fig. 25).

DisCUSIÓN: En la genitalia masculina se distingue de $H$. (H.) subdesertica n. sp. y $H$. (H.) divergens n. sp. porque el gonocoxito carece de abultamientos caudales (figs. $6,7,9$ ) y de procesos laterales membranosos en la hipovalva (figs. 6, 8, 10). En la genitalia interna femenina se distingue de $H$. (H.) subdesertica n. sp. en que el ductus receptaculi es muy largo, filamentoso y formando un ovillo (fig. 20) y no corto y festoneado (fig. 21).

\section{Harraphidia (Harraphidia) subdesertica Monserrat \& Papenberg n. sp.}

Material Estudiado: Tipo: ESPAÑA: Almería: Níjar, Carretera de Níjar, 30SWF7085, 350 m, 23.IV.1988, 1 ○' (a*, $\left.\sigma^{*}\right)$, M. A. Zarazaga (MNCN).

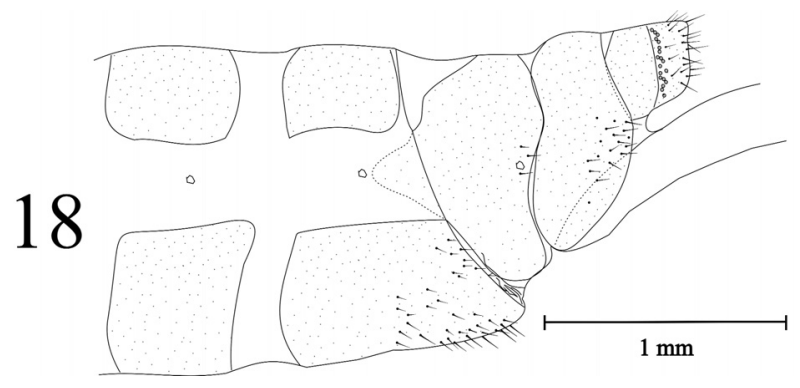

Figs. 17-19.- Genitalia (ㅇ ) en vista lateral de: 17) Harraphidia (H.) harpyia*. 18) Harraphidia (H.) subdesertica n. sp. 19) Harraphidia (F.) laufferi*. Ep: ectoprocto, Es: espiráculo, S: esternito, Sg 7/8: intersegmento 7/8, T: terguito, Tr: tricobotrios. O: ovopositor. * De H. Aspöck et al. (1991).

Figs. 17-19.- Genitalia (ㅇ) in lateral view of: 17) Harraphidia (H.) harpyia*. 18) Harraphidia (H.) subdesertica n. sp. 19) Harraphidia (F.) laufferi*. Ep: ectoproct, Es: espiracle, S: esternite, Sg 7/8: intersegmentum 7/8, T: terguite, Tr: trichobotria. O: ovopositor. * From H. Aspöck et al. (1991).

Paratipos: ESPAÑA: Almería: Níjar, Carretera de Níjar, 30SWF7085, 350 m, 23.IV 1988, $30^{7}$ o $^{7} / 1$ 우 (a*, ㅇ *), M. A. Zarazaga (MNCN). Cádiz: La Almoraima, Castellar, 30STF8117, 45 m, 8.IV.03, 1 오, J. de Ferrer (MNCN).

DiAGNOSIS: Especie con vena suplementaria entre la Costa y el Radio (fig. 2). En el $\sigma^{7}$ la hipovalva está ensanchada en la zona media y sus procesos laterales están orientados en dirección a la línea media (fig. 8). En la $ᄋ$ el ductus receptaculi es festoneado, proporcionalmente corto y sin tener forma de ovillo, y porta pliegues y espinas internas (fig. 21).

\section{DESCRIPCIÓN:}

Morfología eXTerna: Se trata de una especie de tamaño pequeño. La longitud del ala anterior mide entre 6.5-7 mm en el $\sigma^{7}$ (fig. 2) y entre 6-6.5 $\mathrm{mm}$ en la ㅇ. La cabeza es de coloración negra. El clípeo es pardo oscuro y el labro ocre. El escapo, pedicelo y flagelo son pardos. El pronoto se encuentra bordeado por una banda parda. En los apéndices locomotores las cóxas, trocánteres y fémures son pardos, siendo el resto de los segmen- 

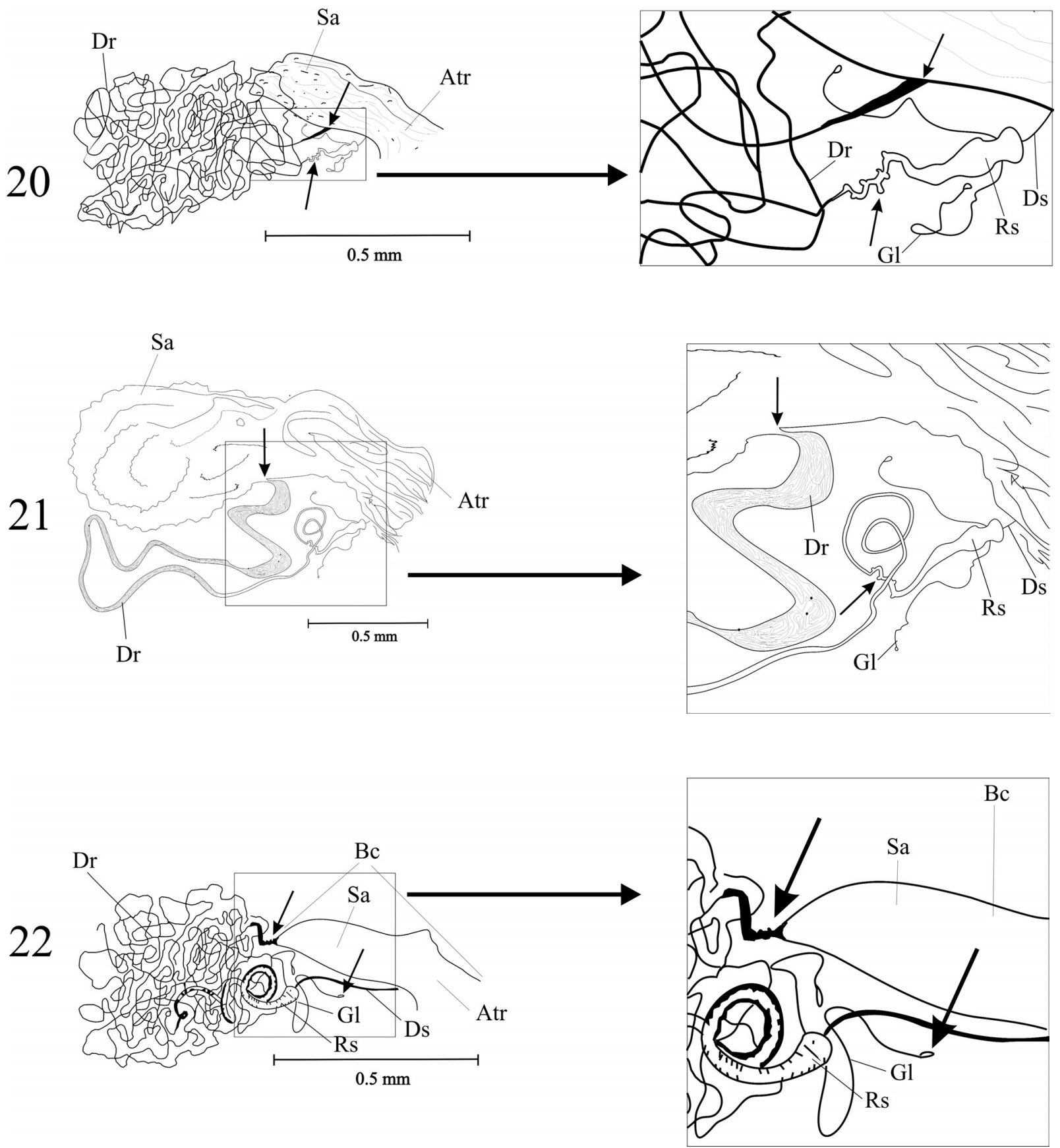

Figs. 20-22.- Genitalia interna ( ㅇ ) vista lateral, ampliada zona del ductus receptaculi y receptáculo seminal de: 20) Harraphidia (H.) harpyia*. 21) Harraphidia (H.) subdesertica n. sp. 22) Harraphidia (F.) laufferi*. Atr: atrio de la bolsa copuladora, Bc: bolsa copuladora, Dr: ductus receptaculi, Ds: ductus seminis, Gl: glándulas accesorias, Rs: receptáculo seminal, Sa: sáculo de la bolsa copuladora. * Adaptado de H. Aspöck et al. (1991).

Figs. 20-22.- Internal genitalia ( ㅇ ) lateral view, with magnification of the ductus receptaculi and seminal receptaculi area of: 20) Harraphidia (H.) harpyia*. 21) Harraphidia (H.) subdesertica n. sp. 22) Harraphidia (F.) laufferi*. Atr: copulatrix bursa atrium, Bc: copulatrix bursa, Dr: ductus receptaculi, Ds: ductus seminis, Gl: accesory glands, Rs: seminal receptaculi, Sa: copulatrix bursa saculus. * Adapted from H. Aspöck et al. (1991). 
tos ocres. La venación alar es, casi en su totalidad, parda, siendo en el primer tercio basal del ala posterior de coloración ocre. Entre la Costa y el Radio hay una vena transversal al igual que entre la Subcosta y el Radio (fig. 2). La coloración de los terguitos y esternitos es parda.

Segmentos Genitales: En el $\sigma^{7}$, la hipovalva es par y presenta dos procesos anteriores que portan espinas y que convergen en los extremos hacia la línea media (fig. 8), en su zona media la hipovalva posee un ensanchamiento globoso, ovoide, levemente recurvado hacia arriba en sus extremos laterales y sus escleritos la recorren en toda su longitud divergiendo en su extremo caudal (fig. 8), sus procesos caudales son marcadamente convergentes hacia su extremo. En la $\odot$ el intersegmento $7 / 8$ es corto y membranoso (fig. 18). El ductus receptaculi parte de la zona ventral del sáculo, es festoneado, no muy largo y no tiene forma de ovillo (fig. 21), su mitad basal posee su membrana interna muy plegada y rugosa, y porta diminutas espinas, su mitad distal, en cambio, es muy lisa y de menor diámetro, presentando unos lóbulos verrugosos en su región terminal (fig. 21), en su tramo final su conducto aumenta de luz formando el receptáculo seminal que posee un par de glándulas largas y dilatadas en sus extremos (fig. 21).

Biología: Su biología es desconocida. Se ha hallado en similares medios que Harraphidia lauffe$r i$, aunque con muy escasa y baja amplitud altitudinal (45-350 m). Por las características ambientales de las zonas donde ha sido hallada, parece poseer una marcada preferencia por los medios xerotérmicos.

Fenología: Su fenología es desconocida. La captura de los ejemplares hasta ahora conocidos se circunscriben al mes de Abril.

Distribución: España. Elemento faunístico estacionario, monocéntrico y xero -mediterráneo. Es de suponer que la distribución se limita exclusivamente a la zona árida del S/S. E. de la Península Ibérica y, por su carácter xerotermo, quede circunscrita a estas zonas ibéricas de clima subdesértico y subtropical (fig. 25).

VARIABILIDAD: En el ejemplar de La Almoraima, Castellar (Cádiz) aparece en ambas alas una vena transversal entre la Costa y el Radio, y dos entre la Subcosta y el Radio.

DISCUSIÓN: Especie halopátrida y muy próxima a Harraphidia (H.) divergens $\mathbf{n}$. sp. al poseer semejante venación alar (figs. 2, 3) y existir un gran parecido en la genitalia del $\sigma^{7}$, sobre todo en lo que respecta a la presencia de procesos membranosos en la hipovalva (figs. 8, 10), pero difieren en su expansión y desarrollo y en la orientación de sus procesos y en la forma caudal de la propia hipovalva. También los procesos ventrales de los gonocoxitos están algo menos desarrollados que en $H$. (H.) divergens $\mathbf{n}$. sp. (figs. 7, 9).

DeRIVATIO NOMINIS: Denominamos así esta especie en reconocimiento al valor ecológico del SE árido de la Península Ibérica, como el mejor y más importante medio desértico-subdesértico del Continente Europeo, que tantas novedades neuropterológicas ha aportado a la fauna europea y mundial y que tan amenazado se halla por la especulación urbanística y el desarrollo no sostenible.

\section{Harraphidia (Harraphidia) divergens Monserrat \& Papenberg n. sp.}

= Harraphidia harpyia Steinmann, 1963: Monserrat et al., 1991: 102 (dis, ie).

Material Estudiado: Tipo: MARRUeCOS: Al Hoceima:

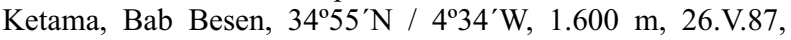
sobre Cistus ladanifer, $10^{7}\left(\mathrm{a}^{*}, \sigma^{*}\right)$, V. J. Monserrat (VM).

Paratipos: MARRUECOS: Al Hoceima: Ketama, Bab Besen, $34^{\circ} 55^{\prime} \mathrm{N} / 4^{\circ} 34^{\prime} \mathrm{W}, 1.600 \mathrm{~m}, 26 . \mathrm{V} .87$, sobre Cistus ladanifer, $2 \sigma^{7} \sigma^{7}$, V. J. Monserrat (VM). Oujda: Sidi Bou Houria, $34^{\circ} 44^{\prime} \mathrm{N} / 2^{\circ} 22^{\prime} \mathrm{W}, 825 \mathrm{~m}, 20$. V.87, sobre Quercus rotundifolia, $10^{7}$, V. J. Monserrat (VM). Taounate: Taounate El Kchour, $34^{\circ} 42^{\prime} \mathrm{N} / 4^{\circ} 35^{\prime} \mathrm{W}, 920 \mathrm{~m}, 20 . \mathrm{V} .87$, sobre Erica arborea, $10^{\circ}$, V. J. Monserrat (VM).

Diagnosis: Especie con vena suplementaria entre las Costa y el Radio (Fig. 3). En el ơ la hipovalva presenta unos procesos digitiformes portadores de espinas y orientados divergentemente hacia fuera (fig. 10). La $ᄋ$ es desconocida.

\section{DESCRIPCIÓN:}

Morfología EXTERna: Es una especie de tamaño pequeño, la longitud del ala anterior mide: 6-8 $\mathrm{mm}$ en el $\sigma^{\pi}$ (fig. 3). La cabeza es de coloración negra. El clípeo y el labro son pardos. El escapo, pedicelo y flagelo son pardo oscuro. El pronoto se encuentra bordeado por una banda ancha parda amarillenta. La coxa I es parda y amarilla anteriormente, las coxas II y III son pardas, el resto de los segmentos son ocres. La venación alar es casi en su totalidad parda, pudiendo ser la Costa, Subcosta y partes del Radio de coloración amarillenta. La región ocupada por el Radio en el ápice alar presenta una vena bifurcada y otra no (fig. 3). Entre la Costa y el Radio hay una vena transversal al igual que entre la Subcosta y el Radio. 

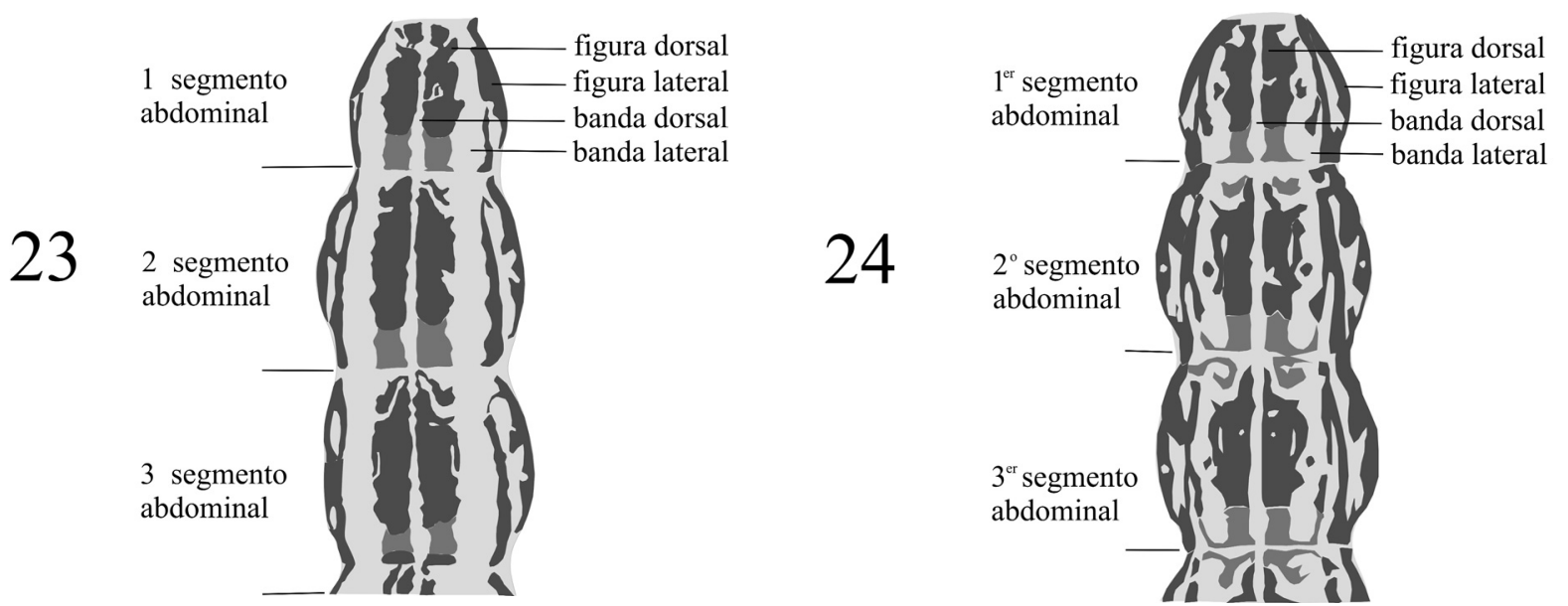

Figs. 23-24.- Vista dorsal de los tres primeros segmentos del abdomen de las larvas de: 23) Harraphidia (H.) harpyia. 24) Harraphidia (F.) laufferi. Adaptado de H. Aspöck et al. (1991).

Figs. 23-24.- Dorsal view of the first three abdominal segments of the larvae of: 23) Harraphidia (H.) harpyia. 24) Harraphidia (F.) laufferi. Adapted from H. Aspöck et al. (1991).

Segmentos genitales: El $\sigma^{7}$, dentro del modelo existente en Harraphidia s. str., presenta el $8^{\circ}$ esternito muy estrecho (fig. 9) en comparación con el $8^{\circ}$ terguito. El $9^{\circ}$ terguito se alarga dorso-ventralmente siendo lateralmente estrecho. El $9^{\circ}$ esternito está representado por una banda muy delgada pero sin el proceso central fino dirigido hacia la zona caudal. Los gonocoxitos se alargan en dirección dorsoventral presentando un apodema del estilo largo que divide al gonocoxito en una región dorsal pequeña $\mathrm{y}$ una ventral grande que presenta un abombamiento en dirección ventro-caudal (fig. 9). Los estilos bifurcados son grandes. La hipovalva es par, sus escleritos presentan dos procesos digitiformes y divergentes que portan pequeñas espinas (fig. 10), no posee ensanchamiento globoso medio y sus escleritos son rectos y estrechos y forman dos bandas que la recorren en toda su longitud, no divergiendo en su extremo caudal (fig. 10). La 우 es por el momento desconocida.

Biología: Su biología es desconocida. Se ha citado en medios térmicos similares a otras especies aquí citadas. Los ejemplares conocidos han sido capturados sobre fanerófitos y nanofanerófitos de carácter mediterráneo (ver material tipo). Como la otra especie marroquí, también parece estar asociada a medios montanos (600-1.600 m).

Fenología: Su fenología es desconocida. Los ejemplares conocidos han sido capturados en el mes de Mayo.
Distribución: Marruecos (fig. 25). Elemento faunístico estacionario, monocéntrico, mauritánico. $\mathrm{Su}$ distribución es escasamente conocida. Es posible que a ella pertenezca alguna de las citas de Harraphidia (H.) harpyia (Steinmann, 1963) n. comb. y así ocurre con las de Monserrat et al. (1991).

DERIVATIO NOMINIS: Denominamos así esta especie por la orientación divergente de los procesos laterales de la hipovalva en los $\sigma^{7} \sigma^{7}$.

Harraphidia (Flavoraphidia) laufferi (Navás, 1915) n. comb.

Raphidia laufferi Navás, 1915

Navás, 1915a: 867 (d), 863, 868, 869 (a), $868\left(\sigma^{7}\right), 867-869$ (mf), 869, 871-874 (dis). Martín \& Izquierdo, 2006: 134 (lst).

Raphidilla laufferi (Navás, 1915)

Bohigas \& Sánchez, 1917: 319 (dis). Navás, 1919: 50 (c), 59 (a), 59-60 (mf), 59 ( (7), 60 (dis). Navás, 1921: 49 (dis). Navás, 1923: 71 (c), 72 (dis). Navás, 1928: 21 (c), 28-29 (a, mf, ơ). Lestage, 1928: 31 (c). Navás, 1933: 121 (lst). Navás, 1934a: 6 (dis). Navás, 1934b: 31 (c), 38-39 (mf), 39 (a, dis, O'). Steinmann, 1964: 216 (a), 217 (c).

Raphidia (Flavoraphidia) laufferi Navás, 1915

H. Aspöck \& U. Aspöck, 1968: 61 (a, mf, ○', 우, tx). H. Aspöck et al., 1980a: - tomo I: 39 (c), 117 (bio, tx), 118, 351 - tomo II: 165 (a), 252 (dis). Díaz Aranda \& Monserrat, 1988a: 215 (dis). Díaz Aranda \& Monserrat, 1988b: 114 (dis). Harraphidia laufferi (Navás, 1915)

H. Aspöck, 1990: 53, 57 (dis). H. Aspöck et al., 1991: tomo I: $129,353,576,577$ (dis), 130 (c), 349, 353, 355 (mf), 
350, 353 (a), 351, 353 ( ᄋ ), 353 (1, O'), 614 (1st) - tomo II: 29-31 (mf), 50-51 (우), 74 (a), 174 ( (7), 270 (우), 343, 354 (1), 487 (dis). H. Aspöck \& Hölzel, 1996: 44 (dis). H. Aspöck et al., 2001: 51 (tx), 51, 332, 338 (dis).

= Raphidia fuentei Navás, 1915

Navás, 1915a: 869 (d), 869-871 (mf), 870-871 (a), 871 (dis, $\left.\sigma^{7}\right)$. Fuente y Morales, 1929: 168-169 (mf), 169 (a). H. Aspöck et al., 1980a: 117 (sin).

$=$ Raphidilla fuentei (Navás, 1915)

Navás, 1919: 50 (c), 60-61 (a, mf), 60 ( $\left.0^{7}\right), 61$ (dis). Navás, 1923: 71 (c), 72 (dis). Navás, 1928: 21 (c), 29-31 (mf), 30 (ơ), 30-31 (a), 31 (dis). Lestage, 1928: 31 (c). Navás, 1933: 121 (lst). Navás, 1934a: 6 (dis). Navás, 1934b: 31 (c), 40 (a, mf, ơ ), 41 (dis). Steinmann, 1964: 216 (a), 217 (c).

Material Estudiado: ESPAÑA: Almería: El Ejido, Punta del Sabinar, 30SWF2660, $88 \mathrm{~m}, 12 . \mathrm{IV} .1992$, sobre Pistacea, $20^{7} \sigma^{7} / 2$ 우 오, V. J. Monserrat (VM). Níjar, carretera de Nijar, 30SWF7085, $350 \mathrm{~m}, 23 . \mathrm{IV} .1988,1$ 우, M. A. Zarazaga (MNCN). Tabernas, Sierra de Alhamilla, 30SWG5400, 25.III.1960, $400 \mathrm{~m}, 10^{7} / 3$ 우 우, Suárez (MNCN). Turrillas, 30SWF6598, 30.V.1989, $800 \mathrm{~m}$, sobre Olea europaea, $20^{7} \sigma^{7} / 1$ ㅇ , A. Baz (VM). Ávila: La Cañada, 30TUK7395, 1.360 m, 9.VI.1991, 1 오, A. Arillo (VM). Cádiz: Alcalá de los Gazules, 30STF5538, 120 m, 2.VI.1990, sobre Genista tridentata, $20^{7} \sigma^{7}$, L. Díaz Aranda (VM). Caños de Meca, 30SQA6608, 20 m, 22.V.2004, sobre Olea europaea, 1 우, V. J. Monserrat (VM). Cerro Alcántara, 30STF3364, $152 \mathrm{~m}$, 23.V.1977, sobre Pistacea, 1 우, V. J. Monserrat (VM). Facinas, 30STF5703, $40 \mathrm{~m}, 1 . \mathrm{V} .2005$, sobre Olea europaea, $7 \sigma^{7} \sigma^{7} / 12$ 우 오, V. J. Monserrat (VM). Jerez de la Frontera, 29SQA5664, 11.V.1991, 1 ㅇ , A. Arillo (VM), 12.V.1991, 10', A. Arillo (VM). La Almoraima, Castellar, 30STF9790, 45 m, 8.IV.2003, $10^{\pi}$, J. de Ferrer (MNCN). Olvera, 30STF9790, $643 \mathrm{~m}$ 28.IV.1986, $10^{\pi / 1}$ 우, M. Baena (VM). Punta Carnero, 30STE8195, $6 \mathrm{~m}, 12 . \mathrm{V} .2002,20^{\top} \sigma^{\top} / 2$ 우 우, J. de Ferrer (MNCN). Tarifa, Los Lances, 30STE6390, 0 m, 9.V.1991, $10^{7} / 10^{2}$, J. de Ferrer (MNCN). Puerto Perales, 29TPE9657, 900 m, 15.V.1980, sobre Pinus pinaster, 2 우 우, V. J. Monserrat (VM). Córdoba: Los Pedroches, 30SUH3285, $530 \mathrm{~m}$, 1.V.2000, sobre Olea europaea, $10^{7}$, leg. ? (EEZ). Granada: Arenales, 30SVG43, 783 m, 1.V.1999, sobre Olea europaea, $40^{7} \sigma^{7} / 2$ 우 우, leg. ? (EEZ). Colomera, 30SVG3636, $737 \mathrm{~m}$, 1.V.1999, sobre Olea europaea, 2 오 오, leg. ? (EEZ), 1.VI.1999, $20^{7} \sigma^{7}$, leg. ? (EEZ). Deifontes, 30SVG5130, 737 m, 1.V.1999, sobre Olea europaea, $130^{7} \sigma^{x} / 8$ 우 우, leg. ? (EEZ), 1.VI.1999, sobre Olea europaea, $20^{7} \sigma^{7}$, leg. ? (EEZ). Jorairatar, 30SVF9086, $800 \mathrm{~m}, 1 . V .1986$, sobre Populus sp., $10^{7} / 2$ 우 우, V. J. Monserrat (VM). Loja, 30SVG9714, $550 \mathrm{~m}$, 1.VI.1986, sobre Celtis australis, $10^{7 / 1}$ ㅇ , leg. ? (VM). Puerto de Onitar, 30SVG4544, 900 m, 1.VI.1986, sobre Olea europaea, $10^{7} / 1$ 오, leg. ? (VM), 1.VII.1986, sobre Olea europaea, 1 o, V. J. Monserrat (VM). Venta del Chaleco, 30SVF8981, 1.200 m, 2.VI.1986, 2 우 우, V. J. Monserrat (VM). Zaidín, Parque de Invierno, 30SVG4612, 22.VI.1982, 10", M. Campos (VM). Zujar, 30SWG1355, $761 \mathrm{~m}$, 19.IV.1981, sobre Pinus halepensis, $10^{7} / 1$ ㅇ․ V. J. Monserrat (VM). Valdenoches, 30TVL9203, 800 m, 14.VI.1984, sobre Pinus halepensis, 1 운, V. J. Monserrat (VM), 21.V.1988, sobre Pinus halepensis, $10^{0}$, F. Marín (VM). Huelva: Aracena, 29SQB1496, 732 m, 19.V.1977, 1 우, V. J. Monserrat (VM). Coto de Doñana, 29SQA3090, 6.VI.1983, 1 ㅇ, C. Montes (VM). Jaén: Campo
Redondo, 30SVH9936, 500 m, 4.VI.1986, sobre Pistacia lentiscus, 1 오, V. J. Monserrat (VM), sobre Olea europaea, $10^{7}$, V. J. Monserrat (VM). Collado de los Jardines, 30SVH5648, 850 m, 2.VI.1989, sobre Pistacea, 1 오, A. Baz (VM), 20.VI.2004, $600 \mathrm{~m}$, sobre Juniperus oxycedrus, 10", V. J. Monserrat (VM). La Aliseda, 30SVH4943, 700 m, 31.V.1986, sobre Quercus ilex, $10^{7} / 1$ 일. leg. ? (VM). Puerto de Despeñaperros, Salto del Fraile, 30SVH5548, $600 \mathrm{~m}$, 31.V.1986, sobre Quercus ilex, 10", V. J. Monserrat (VM), sobre Juniperus oxycedrus, $10^{\prime}$, V. J. Monserrat (VM), sobre Fraxinus, 1 오 , V. J. Monserrat (VM). Río Despeñaperros, Santa Elena, 30SVH54, 742 m, 20.VI.2004, sobre Cistus ladanifer, $10^{7}$, V. J. Monserrat (VM). Sierra de Cazorla, Embalse Tranco de Beas, 30SWH1825, $650 \mathrm{~m}$, 2.VII.1978, encinar, pinar, 1 ㅇ , R. Outerelo (VM). Sierra Magina, 30SVG57, 1.VI.2000, sobre Olea europaea, $20^{7} \sigma^{7} / 9$ ㅇ ㅇ, leg. ? (EEZ). Viñas, 30SVH1622, $600 \mathrm{~m}, 30 . \mathrm{V} .1986$, sobre Pinus pinea, $10^{\pi} / 2$ ㅇ ㅇ , leg. ? (VM). Madrid: Alpedrete, 30TVL1301, 900 $\mathrm{m}$, 28.VI.1975, 1 ㅇ, V. J. Monserrat (VM). Aravaca, 30TVK3479, 650 m, 13.VI.1991, 1 오, V. J. Monserrat (VM). Batres, 30TVK2151, 600 m, 15.V.1978, sobre Olea europaea, $10^{7}$, V. J. Monserrat (VM). Boadilla del Monte, 30TVK2573, 750 m, 10.V.1996, 10", D. Papenberg (VM), 25.VI.2002, 10", D. Papenberg (VM), 16.VI.2002, $10^{7}$, D. Papenberg (VM). Cantoblanco, Universidad Autónoma de Madrid, 30TVK4188, 678 m, 1998, 107, E. García Barros (UAM). Casa de Campo, 30TVK3675, $650 \mathrm{~m}, 25 . \mathrm{V} .1972,10^{7}$, R. Outerelo (VM), 17.V.1974, $10^{7} / 1$ ㅇ, V. J. Monserrat (VM), 26.V.1985, 1 ㅇ , M. Candela (UAM). Cercedilla, 30TVL1010, $1.188 \mathrm{~m}$, 13.VI.1982, 10", R. Outerelo (VM). La Cabezuela, 20.VII.1973, 10", V. de Paz (VM). Ciudad Universitaria, 30TVK3877, 700 m, 1.VI.1971, 10" S. Arnaiz (VM), 8.V.1972, 1 o , J. Hernández (VM), 9.V.1972, $20^{7} \sigma^{7} / 1$ ㅇ, J. Calle (VM), 17.V.1972, $10^{7} / 1$ 우, V. J. Monserrat (VM), 1.VI.1976, 1 o , V. J. Monserrat (VM), 16.V.1978, hierba, $30^{\pi} \sigma^{\pi / 8}$ 우 우, V. J. Monserrat (VM). El Escorial, 30TVK0293, 2 ㅇ ㅇ, Lauffer (MNCN), La Herrería, 30TVK0292, 1.000 m, 24.II.1976, sobre Quercus suber, 1 larva, R. Outerelo (VM). El Pardo, 30TVK3481, 620 m, 14.V.1971, $10^{7}$, J. Martín (VM). El Ventorrillo, 30TVL1312, 1.480 m, 20.VII.1989, 1 오, J. L. Nieves \& Rey (MNCN), 11.VII.1991, 1오, A. Garrido (MNCN). Hoyo de Manzanares, 30TVK2397, $950 \mathrm{~m}$, 1.VII.1975, 1 ㅇ , A. Soler (VM), 23.VI.1980, sobre Juniperus communis, 1 \& , V. J. Monserrat (VM). La Jarosa, Guadarrama, 30TVL0603, $1.000 \mathrm{~m}, 26$.VI.1975, sobre hierba, 1 오 , V. J. Monserrat (VM), 12.VII.1976, sobre Lavandula, 1.100 m, 1 울, J. J. Presa (VM), 24.II.1976, en hojarasca de Cistus ladanifer, 1 larva, R. Outerelo (VM). Madrid, $10^{7}$, R. Flores (VM), 1 오, G. Mercet $(\mathrm{MNCN})$. La Moraleja, 30TVK4784, $650 \mathrm{~m}$, 22.VI.1982, 1 으, M. Candela (MNCN). Puerta de Hierro, 30TVK7938, 20.V.1982, 1 오, M. Candela (MNCN), 24.V.1985, 2 우 우, M. Candela (MNCN). Puerto de la Cruz Verde, 30TUK9790, 1.600 m, VII.1975, $10^{7}$, V. J. Monserrat (VM). Puerto de los Leones, Vía Crucis, 30TVL0305, $1.600 \mathrm{~m}$, 2.X.1975, sobre Cistus, 1 larva, R. Outerelo (VM). Teleférico, 30TVK3674, $650 \mathrm{~m}, 1$. VI.1975, $10^{7}$, M. Candela (MNCN). Villalba, 30TVK1699, 1.108 m, 12.IV.1980, sobre Cistus, 1 오 , P. Garrumi (VM). Málaga: Benadalid, 30STF9754, $690 \mathrm{~m}$, 1.VI.1990, sobre Quercus rotundifolia, 1.VI.1990, 10", A. Baz (VM). Estepona, 30SUF0733, $21 \mathrm{~m}, 31 . \mathrm{V} .1989$, sobre Adenocarpus decorticans, $10^{7}$, A. Baz (VM). Ronda, Sierra de las Nieves, 30SUF1964, 21.VI.1997, 10', J. De Ferrer 
(MNCN). Villanueva del Rosario, 30SUF7895, $697 \mathrm{~m}$, 14.V.1988, 3 우 우, M. A. Zarazaga $(\mathrm{MNCN})$. Murcia: Cehegín, 30SXH0417, 560 m, 10.VI.1982, sobre Dorycnium pentaphyllum, 1 오, V. J. Monserrat (VM). Sierra de Espuña, 30SXG29, 20.VI.1973, $10^{\pi}$, leg. ? (BM). Salamanca: Hinojosa del Duero, 29TPF8539, $601 \mathrm{~m}, 19 . \mathrm{IV} .1980,10^{7}$, J. Pedrero (VM). La Madroñera, 29TPF9417, $700 \mathrm{~m}$, 28.VI.1980, sobre Quercus ilex, $20^{\prime \prime} \sigma^{\prime \prime}$, V. J. Monserrat (VM). Valdecarpinteros, 29TQF1604, $800 \mathrm{~m}, 30 . \mathrm{V} .1983$, sobre Quercus faginea, 1 오, L. S. Subías, Fernández \& Berzosa (VM), 30.VI.1983, $10^{\pi} / 1$ ㅇ , L. S. Subías, Fernández \& Berzosa (VM). Segovia: Chañe, 30TUL8077, $767 \mathrm{~m}, 8 . \mathrm{V}-$ 3.VII.2000, en trampa de Malaise, $10^{7} / 2$ 우 우, J. F. Gómez (VM). El Espinar, 30TUL9408, $1.191 \mathrm{~m}$, 3.VII.1984, hojarasca de pino + musgo, 1 larva, R. Outerelo (VM). Sevilla: Balneario del Pozo Amargo, 30STF8499, 24.V.1977, 1 오, V. J. Monserrat (VM). Toledo: Mora, 30SVJ3393, $740 \mathrm{~m}$, 14.V.1978, sobre Olea europaea, 1 ㅇ, V. J. Monserrat (VM). Sierra de Pelahustán, 30TUK6348, 4.VI.1978, 700 m, sobre Juniperus communis, 1 오, V. J. Monserrat (VM).

Morfología EXTERnA: Es una especie de tamaño pequeño. La longitud del ala anterior mide entre $5,5-7,8 \mathrm{~mm}$ en el $\sigma^{7}$ y entre $6,5-7,5 \mathrm{~mm}$ en la $ᄋ$. El clípeo y el labro son pardos. El escapo es pardo oscuro y tanto el pedicelo como el flagelo son pardo oscuro. El pronoto en su parte cefálica es pardo y en la caudal negro con un dibujo que puede ir desde el amarillo al pardo, el pronoto se encuentra delimitado por una banda amarilla-pardusca. En los apéndices locomotores las coxas II y III son negras y los fémures III pardos. La coxa I, y los fémures I y II son pardos. El resto de los segmentos son amarilloparduscos. La venación alar es, casi en su totalidad, parda, pudiendo aparecer en la base del ala, venas o parte de venas de coloración ocre. El ala anterior no presenta una vena transversal suplementaria entre la Costa y el Radio (fig. 4). La coloración de los terguitos va desde pardo al negro, con una banda media amarillo-ocre. Los esternitos son pardos con manchas laterales y medianas amarillas.

Segmentos genitales: En el $\sigma^{7}$ los escleritos de la hipovalva, en su zona media, presentan un proceso medio triangular (fig. 12), en su parte final se ensanchan a modo de espátula, convergiendo en su región caudal. El endofalo es muy evidente y el hipandrio interno es grande (figs. 11,12, 16). En la o el intersegmento $7 / 8$ es corto, membranoso con pequeñas arrugas. El ductus receptaculi surge del extremo anterior del sáculo (fig. 22), es muy largo y forma un ovillo. El receptáculo seminal es helicoidal, bien esclerificado y las glándulas anejas finalizan en un pequeño ensanchamiento (fig. 22).

Biología: Parece que el desarrollo transcurre en el sustrato y a partir de crías ex-ovo (no finalizadas) se han registrado periodos de desarrollo al menos de 2 años (H. Aspöck et al., 1991). Hemos hallado sus larvas en hojarasca de alcornoques, jaras, pinos y musgos. Hay citas y ahora mencionamos numerosas capturas de imagos en sotobosques, tanto sobre fanerófitos, como nanofanerófitos, sobre todo de acebuches y también en pinares abiertos (especialmente en pinos y enebros jóvenes) aunque ofrece una amplia gama de substratos vegetales, incluso en setos de jardines y puntualmente sobre hierba. Los biotopos son mediterráneos de tendencia xeroterma. La distribución vertical comprende desde el nivel del mar a los $1.600 \mathrm{~m}$. Hemos observado que esta especie muestra evidentes signos de tener capacidad de agregación, ya que en ocasiones hemos recolectado multitud de individuos machos y hembras en determinadas zonas de umbría en sotobosques donde un volumen de $1-2 \mathrm{~m}^{3}$ de vegetación han sido observados docenas de individuos. En situaciones de estrés, como es la captura de ejemplares y alojamiento temporal de individuos vivos en espacios reducidos, se ha observado la existencia de canibalismo. En algunas localidades del sur de la Península Ibérica es simpátrida de Harraphidia (H.) subdesertica $\mathbf{n}$. sp.

FenOlogía: Debido a su mayor dispersión y a su más amplia y variada versatilidad ecológica, esta especie muestra una extensa distribución anual en la aparición de sus imagos, en función de la latitud y la latitud de sus poblaciones. Registros entre los meses de Marzo y Julio.

DistribuCión: Centro y sur de España y Portugal. Elemento faunístico estacionario, monocéntrico e ibérico, probablemente de carácter atlantomediterráneo, aunque sospechamos que su distribución se limita exclusivamente a la Península Ibérica. La frontera septentrional de su distribución no está claramente delimitada, pero el número de registros en el tercio septentrional es de momento nulo (fig. 26), lo que estaría justificado por su carácter xerotermo.

Discusión: Ya hemos discutido sobradamente las peculiaridades morfológicas de esta especie en relación a las otras especies del género. Por su pequeño tamaño se suele reconocer con facilidad, a primera vista se puede confundir con $H$. (H.) subdesertica $\mathbf{n}$. sp. de la que se distingue con claridad por la presencia en esta última de la vena suplementaria entre la Costa y el Radio (figs. 2, 4) y en el $\sigma^{\top}$ por la forma de la hipovalva (figs. 8, 12). Respecto a la larva, se diferencia de la de $H$. (H.) harpyia por la presencia de una mancha parda aislada dentro de la banda pálida lateral (fig. 24). 

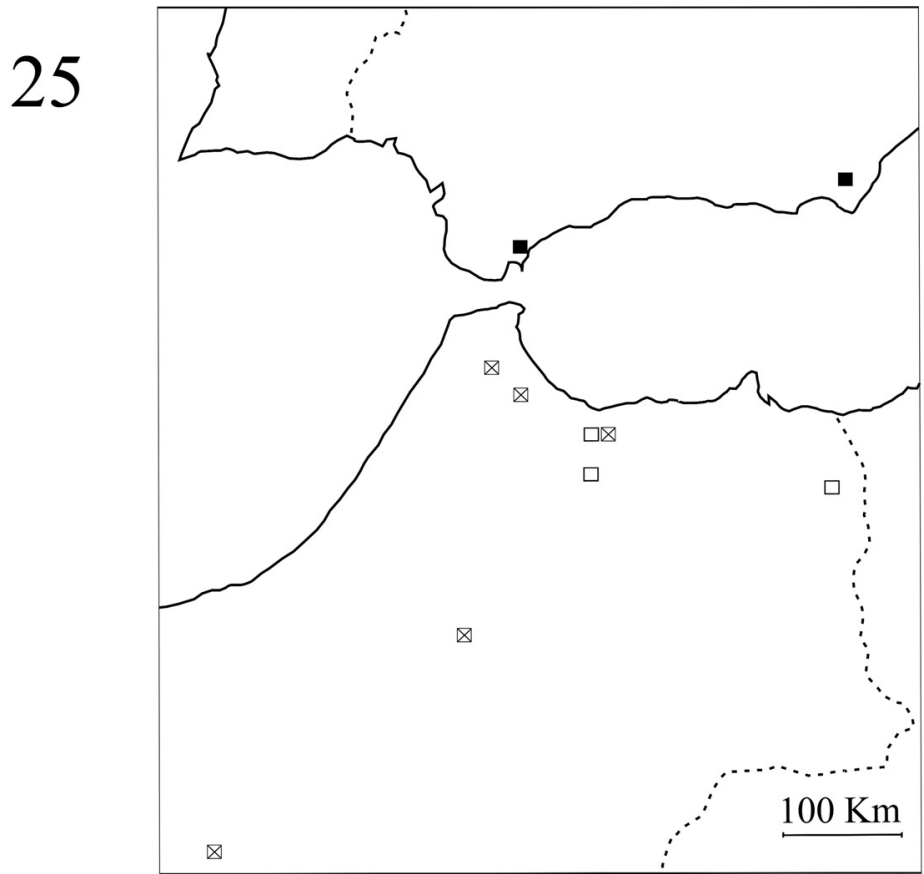

Harraphidia (H.) subdesertica n.sp. Harraphidia (H.) divergens n.sp.

$\otimes$ Harraphidia $(H$.$) harpyia$

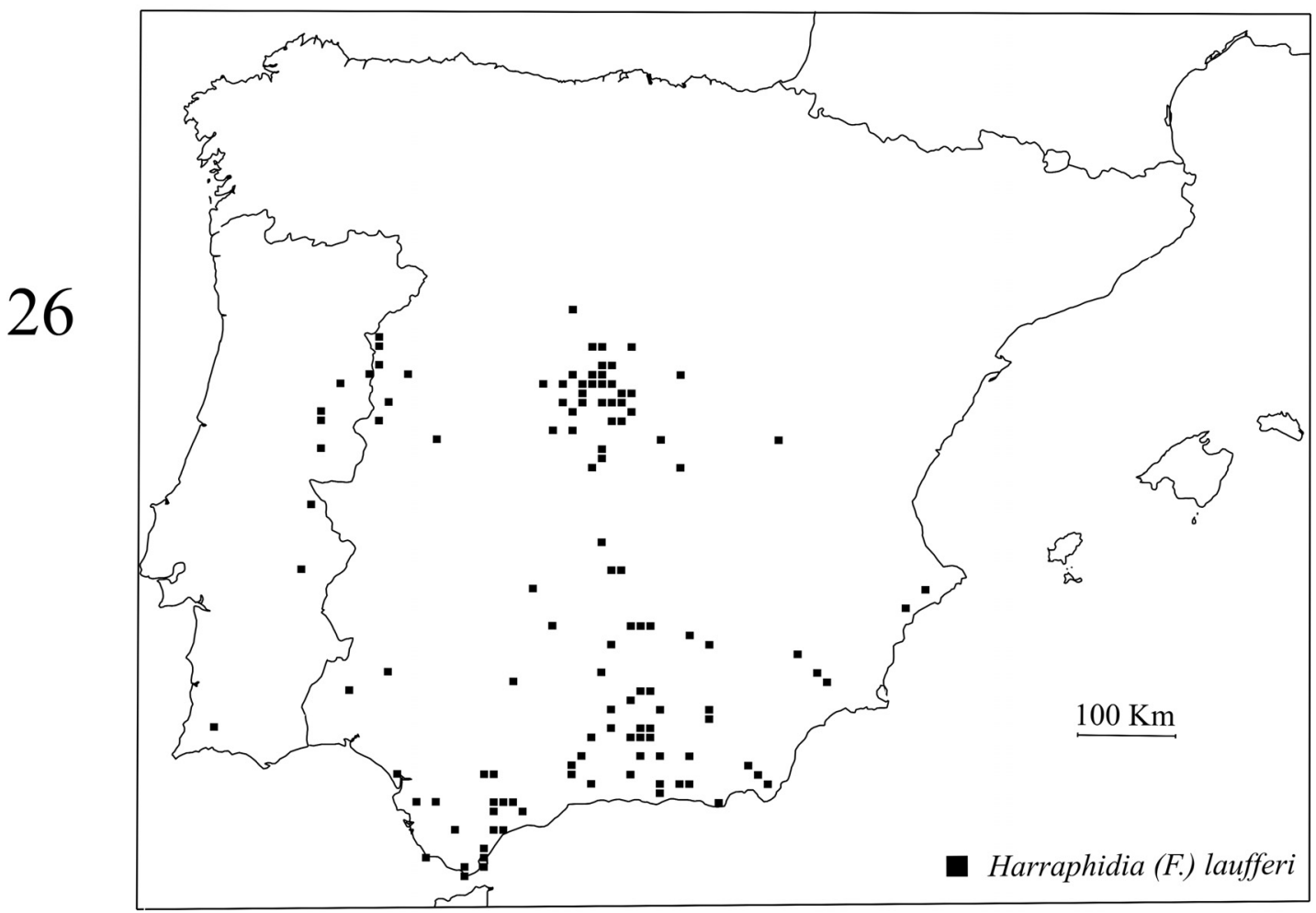

Figs. 25-26.- Mapas de distribución de: 25) Harraphidia (H.) harpyia, Harraphidia (H.) subdesertica n. sp. y Harraphidia (H.) divergens n. sp. 26) Harraphidia (F.) laufferi.

Figs. 25-26.- Distribution maps of: 25) Harraphidia (H.) harpyia, Harraphidia (H.) subdesertica n. sp. and Harraphidia (H.) divergens n. sp. 26) Harraphidia (F.) laufferi. 


\section{4: CONSIDERACIONES BIOGEOGRÁFICAS}

Dada la distribución mayoritariamente (casi exclusivamente) holártica de este orden de insectos (Aspöck et al., 1991, H. Aspöck, 2000, 2002, 2003) parece indudable que la evolución de los rafidiópteros tuvo su origen en el septentrional continente primigenio de Laurasia, y que la presencia de determinados elementos puntuales en continentes Gondwánicos, particularmente el norte de África (donde ya hemos citado los taxones de este orden conocidos) derive de una inicial migración y posterior poblamiento en dirección N-S desde Eurasia. Ya U. Aspöck y H. Aspöck (1994) exponen la teoría que afirma que en el Continente Africano, en sus orígenes, podrían no haber existido rafidiópteros y que fuese posteriormente colonizado desde Europa.

Sin embargo, y como indicábamos al principio, resulta muy paradójica y sorprendente la casi total ausencia de Rafidióperos en todo el amplio tercio norte de la Península Ibérica (ej. fig. 26) donde, salvo algunos escasos elementos que hemos mencionado al principio, existe un vacío casi absoluto de citas sobre estos insectos y que, sin embargo, hubieran podido colonizar toda la Península Ibérica en esta migración N-S. Dados los requerimientos ambientales citados en su desarrollo y su escasa capacidad de vuelo y dispersión, parece que los Pirineos han resultado una barrera casi infranqueable a este flujo N-S de elementos, fueran mayoritariamente paleárticos, paleárticos occidentales o europeos $\mathrm{y}$, por el contrario, la fauna rafidiopterológica ibérica parece, estar muy relacionada con la norte-africana y el género que tratamos es un excelente ejemplo, por lo que sobre este particular conviene realizar algunos comentarios.

Por todo lo anotado en el apartado taxonómico y morfológico, parece lógico pensar en una posición taxonómica más alejada de $H$. (F.) laufferi n. comb. frente a las otras tres especies conocidas y en una mucho más cercana entre $H$. (H.) subdesertica $\mathbf{n}$. sp. y $H$. (H.) divergens n. sp., quedando $H$. (H.) harpyia $\mathbf{n}$. comb. en una posición más intermedia, pero evidentemente más relacionada con las dos últimas especies dentro del mismo subgénero.

Resulta aceptado y aceptable que especies más similares y más relacionadas morfológicamente sean más recientes en su proceso de especiación y viceversa, y que en el caso que nos ocupa, con cuatro especies de las que dos pertenecen a cada uno de los dos continentes (Europa y África), resulta especialmente interesante por la barrera que representa el Estrecho de Gibraltar como generador de un pro- ceso de especiación debido al aislamiento geográfico de poblaciones presunta e inicialmente no separadas.

Se admite que durante el Mesozoico (hace 22565 millones de años) ambos continentes estuvieran en contacto, y cabe la posibilidad de que durante este extenso periodo pudiera haberse realizado una colonización de África desde el norte (Eurasia). Basándose en aspectos morfológicos, es lógico rechazar una colonización tan temprana en este periodo que hubiera dejado huellas en una fauna actual tan homogénea y uniforme como la que existe dentro de este orden de insectos.

Este hecho y el parentesco evidente entre los taxa mencionados exigen asumir que la fauna africana es fruto de una colonización más tardía y reciente durante la Era Terciaria y que posteriormente ha existido un flujo bi-direccional de taxa entre ambos continentes. Usamos como ejemplo en este orden de insectos el género Fibla descrito por Navás (1915b) que sólo es conocido en el Continente Europeo de la Península Ibérica: Fibla (Fibla) hesperica Navás, 1915, de Córcega, Cerdeña y Sicilia: $F$. (F.) maclachlani (Albarda, 1891) y de Creta: Fibla (Reiserella) pasiphae (H. Aspöck \& U. Aspöck, 1971), mientras que en el norte de África alcanza una gran extensión y es colonizado por $F$. (F.) peyerimhoffi (Navás, 1919) de lo que se deduce que ha sido el Continente Africano el origen del género Fibla en el sur del Continente Europeo donde el aislamiento peninsular e insular ha generado nuevas especiaciones.

De igual modo este fenómeno puede explicar una colonización bi-direccional del género Harraphidia desde el Continente Africano a la Península Ibérica y/o viceversa durante las conexiones geográficas existentes durante el Eoceno (hace 40-50 millones de años) o el Mioceno (hace aprox. 6 millones de años). Que el género Harraphidia sólo sea conocido en Europa de la Península Ibérica y en el norte de África (figs. 2526) hace pensar que igual que Fibla su origen se sitúa en el norte de África, y probablemente que desde elementos laurásicos iniciales se haya generado el género Harraphidia durante el Eoceno con elementos comunes en esta zona de contacto entre ambos continentes, evolucionando independientemente tras su posterior separación. Probablemente $H$. (F.) laufferi n. comb. y $H$. (H.) harpyia n. comb. sean resultado de esta primera fragmentación y que durante el Mioceno un nuevo y más reciente flujo $\mathrm{N}-\mathrm{S} / \mathrm{S}-\mathrm{N}$ se produjo, siendo probablemente $H$. $(H$.) divergens n. sp. y $H$. (H.) subdesertica n. sp. el resultado de esta segunda fragmentación. 


\section{Referencias}

AsPÖCK, H., 1986. The Raphidioptera of the World: A review of present knowledge. In: J. Gepp, H. Aspöck \& H. Hölze (eds.). Recent research in Neuropterology. Proceedings of the $2^{\text {nd }}$ International Sympososium of Neuropterology, Hamburg: 15-29.

AspöcK, H., 1990. The Raphidioptera of Africa: a review of the present knowledge (Insecta: Neuropteroidea). In: M. W. Mansell \& H. Aspöck (eds.). Advances in Neuropterology. Proceedings of the 3rd International Symposium on Neuropterology. Berg en Dal, Krüger National Park, 1988: 47-61.

AsPöck, H., 1998. Distribution and biogeography of the order Raphidioptera: updated facts and new hypothesis. Acta Zoologica Fennica, 209: 33-44.

AsPÖCK, H., 2000. Der endekreidezeitliche Impakt und das überleben der Raphidiopteren. Entomologica Basiliensia, 22: 223-233.

AspöcK, H., 2002. The Biology of Raphidioptera: A review of the present knowledge. Acta zoologica hungarica, 48(Suppl. 2): 35-50.

AsPöcK, H., 2003. Kamelhalsfliegen - lebende Fossilien: Eine der Endkreide-Katastrophe entkommende Tiergruppe. Verhandlungen Westdeutscher Entomologentag, 2002: 1-6.

AsPöck, H. \& AsPÖcK, U., 1968. Vorläufige Mitteilung zur generischen Klassifizierung der Raphidiodea (Insecta, Neuroptera). Entomologisches Nachrichtenblatt, Wien, 15: 53-64.

Aspöck, H. \& Aspöck, U., 1969. Die Neuropteren Mitteleuropas. Ein Nachtrag zur «Synopsis der Systematik, Ökologie und Biogeographie der Neuropteren Mitteleuropas». Sonderdruck aus Naturkundliches Jahrbuch der Stadt Linz: 17-68.

AsPÖCK, H. \& AsPÖcK, U., 1971. Ordnung Raphidioptera (Kamelhalsfliegen). In: J-G. Helmcke, D. Starck \& H. Wermuth (eds). Handbuch der Zoologie, 4: Arthropoda: Insecta. Walter de Gruter. Berlin \& New York. 50 pp.

Aspöck, H., Aspöck, U. \& Hölzel, H., 1980a. Die Neuropteren Europas. Eine zusammenfassende Darstellung der Systematik, Ökologie und Chorologie der Neuropteroidea (Megaloptera, Raphidioptera, Planipennia) Europas. 2 Bde. Goecke und Evers. Krefeld. 495 pp., 355 pp.

Aspöck, H., Aspöck, U. \& RAusch, H., 1975. Raphidiopteren - Larven als Bodenbewohner. (Mit Beschreibung der Larven von Ornatoraphidia, Parvoraphidia und Superboraphidia). Zeitschrift für Angewandte Zoologie, 62: 361-375.

AsPöck, H., AsPöck, U. \& RAusch, H., 1980b. Raphidia (Dichrostigma?) santuzza n. sp. - eine neue Kamelhalsfliege aus Kalabrien. Entomologische Zeitschrift, 90: 73-79.
Aspöck, H., Aspöck, U. \& RAusch, H., 1983. Mauroraphidia maghrebina $\mathrm{n}$ gen. $\mathrm{n}$ sp. - eine neue Raphidiiden - Spezies aus dem Atlas. Zeitschrift der Arbeitsgemeinschaft Österreichischer Entomologen, 35: 27-32.

Aspöck, H., Aspöck, U. \& Rausch, H., 1989. The Raphidioptera of the eastern Mediterranean: a zoogeographical analysis. Biologia gallo-hellenica, 15: 67-111.

Aspöck, H., AsPÖck, U. \& Rausch, H., 1991. Die Raphidiopteren der Erde. Eine monographische Darstellung der Systemtatik, Taxonomie, Biologie, Ökologie und Chorologie der rezenten Raphidiopteren der Erde, mit einer zusammenfassenden Ubersicht der fossilen Raphidioteren (Insecta: Neuropteroidea). 2 Bde. Goecke und Evers. Krefeld. 730 pp., 550 pp.

AspöcK, H. \& Hölzel, H., 1996. The Neuropteroidea of North Africa, Mediterranean Asia and of Europe: a comparative review (Insecta). In: M. Canard, H. Aspöck \& M. W. Mansell (eds). Pure and Applied Research in Neuropterology. Proceedings of the Fifth International Symposium on Neuropterology. Cairo, 1994: 31-86.

AsPöcK, H., Hölzel, H. \& AsPöcK, U., 2001. Kommentierter Katalog der Neuropterida (Insecta: Raphidioptera, Megaloptera, Neuroptera) der Westpalärktis. Denisia, 2: 606 pp.

AsPöck, U. \& AsPöcK, H., 1969. Die Raphidiiden Africas (Insecta, Raphidioptera). Annalen des Naturhistorischen Museums in Wien, 73: 161-169.

AsPöcK, U. \& AsPöcK, H., 1994. Paradoxe Verbreitungsbilder von Neuropteroidea (Insecta: Raphidioptera, Neuroptera). Zeitschrift der Arbeitsgemeinschaft Österreichischer Entomologen, 46(1/2): 30-44.

Bohigas, M. \& SÁnchez, A., 1917. Catáleg dels Insectes del Museu pertanyents als ordres: Paraneuroptera Ephemeroptera - Plecoptera - Neuroptera - Megaloptera - Rhaphidioptera - Embioptera - Mecoptera Psocoptera - Trichoptera. Publicacions de la Junta de Ciències Naturals de Barcelona, Anuari, 2 (1 part.): 301-326.

Buresch, I. \& Popov, A., 1973. Ausländische Literatur über die Fauna Bulgariens - Academie Bulgare des Sciences. Bulletin de l'Institut de Zoologie et Musée, 37: 151-156.

Díaz-Aranda, L. M. \& Monserrat, V. J., 1988a. Contribución al conocimiento de los Neurópteros de Granada (Insecta, Neuropteroidea). Actas del Tercer Congreso Ibérico de Entomología, Granada: 211-288.

Díaz-Aranda, L. M. \& Monserrat, V. J., 1988b. Contribución al conocimiento de los neurópteros de Jaén (Insecta, Neuropteroidea). Boletín de la Asociación española de Entomología, 12: 111-123.

ESBEN-PETERSEN, P., 1931. Inventa entomologica itineris Hispanici et Maroccani, quod a. 1926 fecerunt 
Harald et Håkan Lindberg VIII. Neuroptera. Commentationes Biologicae, 3: 1-6.

Fuente y Morales J. M, DE LA, 1929. [Sin título]. Establecimiento tipográfico del Hospicio Provincial. Ciudad Real. 250 pp.

Kovarik, P. W., Burke, H. R. \& Agnew, C. W., 1991. Development and Behavior of Snakefly, Raphidia bicolor Albarda (Neuroptera: Raphidiidae). Southwestern Entomologist, 16(4): 353-364.

Lestage, J. A., 1922. Description d'un Raphidide (Neur.) algérien nouveau. Bulletin de la Société entomologique de France, 1922: 175-178.

Lestage, J. A., 1928. Une Inocellia nouvelle du Maroc et Notes critiques sur les Raphidioidea (Megaloptera). Bulletin de la Société des Sciences Naturelles du Maroc, 8: 26-33.

Martín Alabaladejo, C. \& IzQuierdo Moya, I., 2006. Tipos de especies de insectos descritas de la Comunidad de Madrid, conservados en el Museo Nacional de Ciencias Naturales. Inventario preliminar. Graellsia, 62(número extraordinario): 109-144.

Monserrat V. J., Díaz Aranda, L. M. \& Hölzel, H., 1991. Contribución al conocimiento de los Neurópteros de Marruecos (Insecta, Neuropteroidea). Eos, [1990], 66(2): 101-115.

NAVÁs, L., 1915a. Notas sobre Rafídidos (Ins. Neur.). Revista de la Real Academia de Ciencias Exactas, Físicas y Naturales de Madrid, 13: 860-874.

NAVÁs, L., 1915b. Neurópteros nuevos o poco conocidos. Quinta serie. Memorias de la Real Academia de Ciencias y Artes de Barcelona, 11: 455-480.

NAVÁs, L., 1919. Monografia de l'Ordre dels Rafidiópters (Ins.). Publicacions de l'Institut de Ciències, 1918: 1$90 \mathrm{pp}$.

NAVÁs, L., 1921. Insectos del Escorial recogidos por D. Jorge Lauffer. Boletín de la Sociedad Entomológica de España, IV: 49.
NAVÁs, L., 1923. Sinopsis de los Rafidiópteros (Insectos) de España y Portugal. Asociación Española para el Progreso de las Ciencias, Congreso de Salamanca, Salamanca: 67-73.

NAVÁs, L., 1928. Rafidiópteros de la Península Ibérica. Memorias de la Sociedad Entomológica de España, 2: 5-56.

NAVÁs, L., 1933. La colección de Rafidiópteros (Im.) del Museo de Barcelona. Boletín de la Sociedad Entomológica de España, 16: 120-121.

NaVÁs, L., 1934a. Els Rafidiópters del Museu de Ciéncies Naturals de Barcelona. Treballs del Museu de Ciències Naturals de Barcelona, 11(7): 1-7.

NAVÁs, L., 1934b. Entomología de Catalunya Neurópteros: Rafidiopteros. Arxius de l'Institut de Ciències, 1934: 1-66.

SteInMAnN, H., 1963. Raphidiopterological Studies I. Navasana gen. n. from Hungary, Harraphidia Gen. n. from Morocco, New Lesna NAV. and Subilla NAV. species from Europe. Acta zoologica hungarica, 9: 183-198.

Steinmann, H., 1964. Raphidiopterological studies II. New Raphidia L. and Rhaphidilla Nav. species from Europe and Asia. Acta zoologica hungarica, 10: 199227.

Oswald, J. D. \& PENNY, N. D., 1991. Genus-Group names of the Neuroptera, Megaloptera and Raphidioptera of the world. Occasional papers of the California Academy of Sciences, 147: 1-94.
Recibido, 13-VII-2006 Aceptado, 31-X-2006 Publicado, 29-XII-2006 